\title{
Multivariate measures of skewness for the scale mixtures of skew-normal distributions
}

\author{
Hyoung-Moon $\mathrm{Kim}^{1, a}$, Jun Zhao ${ }^{a}$ \\ ${ }^{a}$ Department of Applied Statistics, Konkuk University, Korea
}

\begin{abstract}
Several measures of multivariate skewness for scale mixtures of skew-normal distributions are derived. As a special case, those of multivariate skew- $t$ distribution are considered in detail. Furthermore, the similarities, differences, and behavior of these measures are explored for cases of some specific members of the multivariate skew-normal and skew- $t$ distributions using a simulation study. Since some measures are vectors, it is better to take all measures in the same scale when comparing them. In order to attain such a set of comparable indices, the sample version is considered for each of the skewness measures that are taken as test statistics for the hypothesis of $t$ distribution against skew- $t$ distribution. An application is reported for the data set consisting of 71 total glycerol and magnesium contents in Grignolino wine.
\end{abstract}

Keywords: scale mixtures, skew-normal, skew- $t$, multivariate skewness

\section{Introduction}

The density function of a multivariate skew-normal distribution (SN) by Azzalini and Capitanio (2014), $\mathbf{z} \sim \mathrm{SN}_{p}(\overline{\mathbf{\Omega}}, \boldsymbol{\alpha})$, is given by

$$
2 \phi_{p}(\mathbf{z} ; \overline{\mathbf{\Omega}}) \Phi\left(\boldsymbol{\alpha}^{\top} \mathbf{z}\right), \quad \mathbf{z} \in \mathbb{R}^{p}
$$

where $\overline{\mathbf{\Omega}}$ is a positive-definite $p \times p$ correlation matrix, $\phi_{p}(\mathbf{z} ; \overline{\mathbf{\Omega}})$ denotes the density function of a $\mathbf{N}_{d}(0, \overline{\mathbf{\Omega}}), \Phi(\cdot)$ is the $N(0,1)$ distribution function and $\boldsymbol{\alpha}$ is a $p$-dimensional vector parameter controlling skewness. The location and scale extension is obtained by

$$
\mathbf{x}=\xi+\omega \mathbf{z},
$$

where $\boldsymbol{\xi}=\left(\xi_{1}, \ldots, \xi_{p}\right)^{\top}, \boldsymbol{\omega}=\operatorname{diag}\left(\omega_{1}, \ldots, \omega_{p}\right), \omega_{i}=\sqrt{\omega_{i i}}$, and $\boldsymbol{\Omega}=\left(\omega_{i j}\right)=\boldsymbol{\omega} \overline{\mathbf{\Omega}} \boldsymbol{\omega}$ is a full rank $p \times p$ covariance matrix. Then the density function of $\mathbf{x}$ is

$$
2 \phi_{p}(\mathbf{x}-\boldsymbol{\xi} ; \boldsymbol{\Omega}) \Phi\left\{\boldsymbol{\alpha}^{\top} \boldsymbol{\omega}^{-1}(\mathbf{x}-\boldsymbol{\xi})\right\}, \quad \mathbf{x} \in \mathbb{R}^{p}
$$

We use $\mathbf{x} \sim \mathrm{SN}_{p}(\boldsymbol{\xi}, \boldsymbol{\Omega}, \boldsymbol{\alpha})$ to indicate that $\mathbf{x}$ has density function (1.1).

Branco and Dey (2001) introduced scale mixtures of skew-normal distribution as:

$$
\mathbf{y}=\boldsymbol{\xi}+W(\eta)^{\frac{1}{2}} \mathbf{x}
$$

\footnotetext{
${ }^{1}$ Corresponding author: Department of Applied Statistics, Konkuk University, 120 Neungdong-ro, Gwangjin-gu, Seoul 05029, Korea. E-mail: hmkim@konkuk.ac.kr
}

Published 31 March 2018 / journal homepage: http://csam.or.kr

(c) 2018 The Korean Statistical Society, and Korean International Statistical Society. All rights reserved. 
Table 1: Special cases of scale mixtures of skew-normal distributions

\begin{tabular}{lcc}
\hline \hline Distribution & $W(\eta)$ & $h(\eta)$ \\
\hline skew- $t$ & $1 / \eta$ & $\frac{(v / 2)^{\frac{v}{2}}}{\Gamma(v / 2)} \eta^{\frac{v}{2}-1} e^{-\frac{v \eta}{2}}, \quad \eta>0$ \\
\hline skew-logistic & $4 \eta^{2}$ & $8 \sum_{k=1}^{\infty}(-1)^{k+1} k^{2} \eta \exp \left(-2 k^{2} \eta^{2}\right), \quad \eta>0$ \\
\hline skew-slash & $1 / \eta^{\frac{2}{q}}, \quad q>0$ & $\eta \sim U(0,1)$ \\
\hline \hline
\end{tabular}

Table 2: Moments of mixing distributions

\begin{tabular}{cc}
\hline \hline \multicolumn{1}{c}{ Distribution } & $c_{m}$ \\
\hline skew- $t$ & $\frac{(v / 2)^{\frac{m}{2}} \Gamma((v-m) / 2)}{\Gamma(v / 2)}, \quad v>m$ \\
\hline skew-logistic & $2^{1+\frac{m}{2}} \Gamma\left(\frac{m}{2}+1\right) \sum_{k=1}^{\infty}(-1)^{k+1} / k^{m}$ \\
\hline skew-slash & $\frac{q}{q-m}, q>m$ \\
\hline finite mixture of skew-normal & $\sum_{j=1}^{n} W\left(\eta_{j}\right)^{\frac{m}{2}} \pi_{j}$ \\
\hline skew-contaminated-normal & $\frac{\pi}{\gamma^{\frac{m}{2}}}+1-\pi$ \\
\hline \hline
\end{tabular}

where $\mathbf{x} \sim \mathrm{SN}_{p}(\mathbf{0}, \boldsymbol{\Omega}, \boldsymbol{\alpha})$, and $\eta$ is a mixing variable with density function $H(\eta)$ and a weight function $W(\eta)$, independent of $\mathbf{x}$. Therefore, the density function of $\mathbf{y}$ is

$$
2 \int_{0}^{\infty} \phi_{p}(\mathbf{y} ; \boldsymbol{\xi}, W(\eta) \mathbf{\Omega}) \Phi\left\{W(\eta)^{-\frac{1}{2}} \boldsymbol{\alpha}^{\top} \boldsymbol{\omega}^{-1}(\mathbf{y}-\boldsymbol{\xi})\right\} d H(\eta)
$$

We indicate $\operatorname{SMSN}_{p}(\boldsymbol{\xi}, W(\eta) \boldsymbol{\Omega}, \boldsymbol{\alpha})$ as scale mixtures of skew-normal distributions (SMSN) with the density function (1.2).

Table 1 summarizes some special cases of scale mixtures of skew-normal distributions along with their mixing density function $h(\eta)$. Furthermore, when the distribution $H$ is a discrete measure on $\left\{\eta_{1}, \eta_{2}, \ldots, \eta_{n}\right\}$ with probabilities $p_{1}, p_{2}, \ldots, p_{n}$, respectively, then the finite mixture of skew-normal distributions is obtained. One special case occurs when $W(\eta)=1 / \eta$ and $H$ is a discrete measure on $\left\{\eta_{1}=\gamma, \eta_{2}=1\right\}$ with probabilities $p, 1-p$, respectively. Then the skew-contaminated-normal distribution is obtained.

The moments of mixing distributions are defined by:

$$
\mathrm{E}\left(W(\eta)^{\frac{m}{2}}\right)=c_{m}, \quad m=1,2, \ldots
$$

Table 2 summarizes the moments of mixing distributions for some special cases of scale mixtures of skew-normal distributions.

When $W(\eta)=1$, which leads to $c_{m}=1$, the scale mixtures of multivariate skew-normal distribution reduce to multivariate skew-normal distribution. The mean of $\mathbf{y} \sim \operatorname{SMSN}_{p}(\boldsymbol{\xi}, W(\eta) \boldsymbol{\Omega}, \boldsymbol{\alpha})$ is

$$
\boldsymbol{\mu}=E(\mathbf{y})=\boldsymbol{\xi}+\sqrt{\frac{2}{\pi}} c_{1} \gamma
$$

and covariance matrix is

$$
\boldsymbol{\Sigma}=\operatorname{Cov}(\mathbf{y})=c_{2} \boldsymbol{\Omega}-\frac{2}{\pi} c_{1}^{2} \boldsymbol{\gamma} \boldsymbol{\gamma}^{\top},
$$


where $\boldsymbol{\gamma}=\boldsymbol{\omega} \boldsymbol{\delta}$ and $\boldsymbol{\delta}=\overline{\boldsymbol{\Omega}} \boldsymbol{\alpha} /\left(1+\boldsymbol{\alpha}^{\top} \overline{\mathbf{\Omega}} \boldsymbol{\alpha}\right)^{1 / 2}$.

Skewness is used to describe a systematic deviation from the symmetry of a univariate distribution. Many measures of skewness have also been developed as described by Benjamini and Krieger (2006). Multivariate skewness measures were developed from different viewpoints for multivariate distributions, because the different directions can be characterized by univariate skewness measures in different ways. Balakrishnan and Scarpa (2012) calculated and compared different multivariate measures of skewness for multivariate skew-normal distribution. Therefore, it is desirable to derive some skewness measures for scale mixtures of multivariate skew-normal distributions that include multivariate skew-normal distribution as a special case. Another important case, a multivariate skew- $t$ distribution, is also obtained by a scale mixtures of multivariate skew-normal distribution. It is therefore meaningful to do a comparison among all multivariate skewness measures for multivariate skew- $t$ distribution and another comparison between multivariate skew- $t$ and skew-normal distributions for each skewness measure in simulation studies. A real data example is helpful to understand how to apply those measures in practice.

This article is organized as follows. Section 2 describes some skewness measures and derive their expressions for the scale mixtures of multivariate skew-normal distribution. A particular case, those of the multivariate skew- $t$ distribution are considered in detail. Furthermore, a simulation study is done in Section 3 using the multivariate skew- $t$ distributions with different parameters. These measures are also calculated using the same location and scale parameters for the case of a multivariate skewnormal distribution. The comparison among the measures is also done simultaneously. In Section 4, hypothesis tests and the performance of all skewness measures in the testing context are discussed. An application study is also given in Section 5 to illustrate how each skewness measure performs in practice. Finally, a short discussion is given.

\section{Some multivariate measures of skewness}

\subsection{Mardia index}

Mardia (1970) introduced one of the popular and commonly used measures of multivariate skewness of an arbitrary $p$-dimensional distribution $F$ with mean vector $\boldsymbol{\mu}$ and covariance matrix $\Sigma$. If $\mathbf{x}=$ $\left(x_{1}, \ldots, x_{p}\right)^{\top}$ and $\mathbf{y}=\left(y_{1}, \ldots, y_{p}\right)^{\top}$ are two independent and identically distributed random vectors from this distribution, the Mardia measure of skewness is defined as

$$
\gamma_{1, p}=\beta_{1, p}=E\left\{\left[(\mathbf{x}-\boldsymbol{\mu})^{\top} \boldsymbol{\Sigma}^{-1}(\mathbf{y}-\boldsymbol{\mu})\right]^{3}\right\} .
$$

Using Theorem 1 of Kim and Kim (2017), they obtained Mardia's measure of multivariate skewness of $\mathbf{y} \sim \operatorname{SMSN}_{p}(\boldsymbol{\xi}, W(\eta) \boldsymbol{\Omega}, \boldsymbol{\alpha})$. Derived measure is equal to (6.20) of Azzalini and Capitanio (2014) who obtained the measure using the canonical form of the scale mixtures of multivariate skew-normal distributions. This measure is

$$
\gamma_{1, p}=\left(\gamma_{1}^{*}\right)^{2}+\frac{3(p-1)}{\sigma_{*}^{2} c_{2}^{2}}\left(c_{3}-c_{1} c_{2}\right)^{2} \frac{2}{\pi} \delta_{*}^{2},
$$

where $c_{i}$ is defined in (1.3),

$$
\gamma_{1}^{*}=\frac{b \delta_{*}}{\sigma_{*}^{3}}\left\{\left(\frac{4}{\pi} c_{1}^{3}-c_{3}\right) \delta_{*}^{2}-3\left(c_{1} c_{2}-c_{3}\right)\right\},
$$




$$
\begin{aligned}
b=\sqrt{2 / \pi}, \sigma_{*}^{2}=c_{2}-b^{2} c_{1}^{2} \delta_{*}^{2}, \delta_{*}=\sqrt{\boldsymbol{\delta}^{\top} \overline{\mathbf{\Omega}}^{-1} \boldsymbol{\delta}}, \text { and } \\
\qquad \delta=\frac{\overline{\boldsymbol{\Omega}} \boldsymbol{\alpha}}{\left(1+\boldsymbol{\alpha}^{\top} \overline{\mathbf{\Omega}} \boldsymbol{\alpha}\right)^{\frac{1}{2}}} .
\end{aligned}
$$

Here the quantities $\gamma_{1}^{*}$ and $\sigma_{*}^{2}$ refer to the unique skewed component of canonical form.

\subsection{Malkovich-Afifi measure}

Kim and Kim (2017) obtained Malkovich-Afifi measure (Malkovich and Afifi, 1973) of multivariate skewness, $\beta_{1}^{*}$, using a similar approach of Balakrishnan and Scarpa (2012) as:

$$
\beta_{1}^{*}=\left(\gamma_{1}^{*}\right)^{2}
$$

where $\gamma_{1}^{*}$ is given in (2.1).

It is well-known that in the multivariate skew-normal distribution Mardia's and Malkovich-Afifi's skewness indices are equal and agree with the square of the skewness of the only skewed component of the canonical form (Balakrishnan and Scarpa, 2012). However, this is not true for the scale mixtures of multivariate skew-normal distributions, where Mardia's measure is different from the square of the skewness of the only skewed canonical variable $z_{1}^{*}$ (Azzalini and Capitanio, 2014). Arevalillo and Navarro (2015) obtained Malkovich-Afifi's measure of multivariate skewness via finding the direction with maximal skewness for a multivariate skew- $t$ distribution for integer degrees of freedom.

\subsection{Balakrishnan-Brito-Quiroz measure}

Balakrishnan et al. (2007) modified the Malkovich-Afifi index to produce a vectorial measure of skewness as

$$
\mathbf{t}=\int_{\emptyset_{p}} \mathbf{u} c_{1}(\mathbf{u}) d \lambda(\mathbf{u})
$$

where $c_{1}(\mathbf{u})=E\left[\left(\mathbf{u}^{\top} \mathbf{z}\right)^{3}\right]$ is a signed measure of skewness of the standardized variable $\mathbf{z}=\mathbf{\Sigma}^{-1 / 2}(\mathbf{y}-$ $E(\mathbf{y}))$ in the direction of $\mathbf{u}$, and $\lambda$ denotes the rotationally invariant probability measure on the unit $p$-dimensional sphere $\emptyset_{p}=\left\{\mathbf{x} \in \mathbb{R}^{p}:\|\mathbf{x}\|=1\right\}$. For obtaining a single measure, they proposed the quantity $Q=\mathbf{t}^{\top} \boldsymbol{\Sigma}_{\mathbf{t}} \mathbf{t}$, where $\mathbf{t}$ is given in (2.3) and $\boldsymbol{\Sigma}_{\mathbf{t}}$ is the covariance matrix of $\mathbf{t}$. However the covariance matrix $\Sigma_{\mathbf{t}}$ depends on the moments of sixth order and for the SMSN case they are not available yet in an explicit form. Replacing $\boldsymbol{\Sigma}_{\mathbf{t}}$ by $\boldsymbol{\Sigma}_{\mathbf{z}}$, we obtain $Q^{*}=\mathbf{t}^{\top} \boldsymbol{\Sigma}_{\mathbf{z}} \mathbf{t}$ which provides a reasonable measure of overall multivariate skewness.

Using the integrals of some monomials over the unit sphere $\emptyset_{p}$, we have

$$
\begin{aligned}
J_{4} & =\int_{\emptyset_{p}} y_{j}^{4} d \lambda(y)=\frac{3}{p(p+2)}, \\
J_{2,2} & =\int_{\emptyset_{p}} y_{j}^{2} y_{i}^{2} d \lambda(y)=\frac{1}{p(p+2)},
\end{aligned}
$$

for $j \neq i, 1 \leq j, i \leq p$. The integrals do not depend on particular choices of $j$ and $i$, hence the $r^{t h}$ coordinate of $\mathbf{t}$ is

$$
T_{r}=J_{4} E\left(Y_{r}^{3}\right)+3 \sum_{i \neq r} J_{2,2} E\left(Y_{i}^{2} Y_{r}\right)
$$


Therefore to obtain $T_{r}$, we need the moments $E\left(Y_{r}^{3}\right)$ and $E\left(Y_{i}^{2} Y_{r}\right)$. Kim and Kim (2017) obtained the following moments:

$$
\begin{aligned}
\mathbf{M}_{3}(\mathbf{y})= & E\left(\mathbf{y} \otimes \mathbf{y}^{\top} \otimes \mathbf{y}\right) \\
= & c_{2}\left[\mathbf{\Omega} \otimes \boldsymbol{\xi}+\boldsymbol{\xi} \otimes \mathbf{\Omega}+\operatorname{vec}(\mathbf{\Omega}) \xi^{\top}\right]+\boldsymbol{\xi} \boldsymbol{\xi}^{\top} \otimes \boldsymbol{\xi} \\
& +\sqrt{\frac{2}{\pi}} c_{3}\left[\boldsymbol{\gamma} \otimes \mathbf{\Omega}+\operatorname{vec}(\mathbf{\Omega}) \boldsymbol{\gamma}^{\top}+\mathbf{\Omega} \otimes \boldsymbol{\gamma}-\boldsymbol{\gamma} \boldsymbol{\gamma}^{\top} \otimes \gamma\right] \\
& +\sqrt{\frac{2}{\pi}} c_{1}\left[\gamma \boldsymbol{\xi}^{\top} \otimes \boldsymbol{\xi}+\boldsymbol{\xi} \boldsymbol{\gamma}^{\top} \otimes \boldsymbol{\xi}+\boldsymbol{\xi} \boldsymbol{\xi}^{\top}, \otimes \gamma\right]
\end{aligned}
$$

where $\gamma=\omega \delta(\boldsymbol{\Omega}=\omega \overline{\mathbf{\Omega}} \omega), \otimes$ and vec are the Kronecker operator and the vec operator respectively. Hence the specific moments can be obtained by

$$
\begin{aligned}
E\left(Y_{r}^{3}\right) & =\mathbf{M}_{3}[(r-1) p+r, r] \\
& =\xi_{r}\left(3 c_{2} \omega_{r r}+\xi_{r}^{2}\right)+\sqrt{\frac{2}{\pi}} c_{3} \gamma_{r}\left[3 \omega_{r r}-\gamma_{r}^{2}\right]+3 \sqrt{\frac{2}{\pi}} c_{1} \gamma_{r} \xi_{r}^{2}, \\
E\left(Y_{i}^{2} Y_{r}\right) & =\mathbf{M}_{3}[(i-1) p+i, r] \\
& =c_{2}\left[2 \omega_{i r} \xi_{i}+\omega_{i i} \xi_{r}\right]+\xi_{i}^{2} \xi_{r}+\sqrt{\frac{2}{\pi}} c_{3}\left[2 \gamma_{i} \omega_{i r}+\gamma_{r} \omega_{i i}-\gamma_{i}^{2} \gamma_{r}\right]+\sqrt{\frac{2}{\pi}} c_{1}\left[2 \gamma_{i} \xi_{i} \xi_{r}+\gamma_{r} \xi_{i}^{2}\right],
\end{aligned}
$$

where $\mathbf{M}_{3}[\cdot, \cdot]$ denotes the elements of matrix $\mathbf{M}_{3}$. Using these moments, we have the elements of $\mathbf{t}$ as

$$
\begin{aligned}
T_{r}= & J_{4} E\left(Y_{r}^{3}\right)+3 \sum_{i \neq r} J_{2,2} E\left(Y_{i}^{2} Y_{r}\right) \\
= & J_{4}\left\{\xi_{r}\left(3 c_{2} \omega_{r r}+\xi_{r}^{2}\right)+\sqrt{\frac{2}{\pi}} c_{3} \gamma_{r}\left[3 \omega_{r r}-\gamma_{r}^{2}\right]+3 \sqrt{\frac{2}{\pi}} c_{1} \gamma_{r} \xi_{r}^{2}\right\} \\
& +3 \sum_{i \neq r} J_{2,2}\left\{c_{2}\left[2 \omega_{i r} \xi_{i}+\omega_{i i} \xi_{r}\right]+\xi_{i}^{2} \xi_{r}+\sqrt{\frac{2}{\pi}} c_{3}\left[2 \gamma_{i} \omega_{i r}+\gamma_{r} \omega_{i i}-\gamma_{i}^{2} \gamma_{r}\right]+\sqrt{\frac{2}{\pi}} c_{1}\left[2 \gamma_{i} \xi_{i} \xi_{r}+\gamma_{r} \xi_{i}^{2}\right]\right\} .
\end{aligned}
$$

\subsection{Isogai measure}

Isogai (1982) introduced another measure of multivariate skewness, given by

$$
S_{I}=\left(\boldsymbol{\mu}-\boldsymbol{M}_{0}\right)^{T} g^{-1}(\boldsymbol{\Sigma})\left(\boldsymbol{\mu}-\boldsymbol{M}_{0}\right),
$$

where $\boldsymbol{M}_{0}$ is the mode of the distribution and $g(\boldsymbol{\Sigma})$ is an appropriate function of the covariance matrix $\Sigma$.

To derive this measure, we need to know the mode of the distribution. However the uniqueness of the mode for the family of scale mixture of skew-normal distributions other than skew-normal and skew- $t$ distributions is an open problem by Capitanio (2012). So we derive the Isogai measure for skew- $t$ distribution by choosing $g(\cdot)$ to be the identity function. This measure is location and scale invariant so by using canonical form of skew- $t$ distribution, we have

$$
S_{I}=\frac{\left(b_{v} \delta_{*}-y_{0}^{*}\right)^{2}}{\frac{v}{v-2}-b_{v}^{2} \delta_{*}^{2}},
$$


where $\delta_{*}=\left(\boldsymbol{\delta}^{\top} \overline{\boldsymbol{\Omega}}^{-1} \boldsymbol{\delta}\right)^{1 / 2}, b_{v}=\{\sqrt{v} \Gamma((v-1) / 2)\} /\{\sqrt{\pi} \Gamma(v / 2)\}$, and $y_{0}^{*} \in \mathbb{R}$ is the unique solution of the equation

$$
y(v+p)^{\frac{1}{2}} T_{1}(w(y) ; v+p)-t_{1}(w(y) ; v+p) v \alpha_{*}\left(v+y^{2}\right)^{-\frac{1}{2}}=0,
$$

where $w(y)=\alpha_{*} y\left((v+p) /\left(v+y^{2}\right)\right)^{1 / 2}, \alpha_{*}^{2}=\delta_{*}^{2} /\left(1-\delta_{*}^{2}\right) \in \mathbb{R}$ and $t_{1}, T_{1}$ are the density function and distribution function of the skew- $t$ distribution, respectively.

Another vectorial measure was given by Balakrishnan and Scarpa (2012), that is, $S_{C}=\boldsymbol{\omega}^{-1}(\boldsymbol{\mu}-$ $\boldsymbol{M}_{0}$ ) is a natural choice to characterize the direction of the asymmetry of multivariate skew-normal distribution. Using the same reasoning for skew- $t$ distribution, this leads to

$$
S_{C}=\left(\sqrt{\frac{2}{\pi}} c_{1}-\frac{y_{0}^{*}}{\delta_{*}}\right) \delta .
$$

Therefore, the direction of $\delta$ can be regarded as a measure of vectorial skewness for multivariate skew- $t$ distribution.

\subsection{Srivastava measure}

Srivastava measure of skewness (Srivastava, 1984) is based on the principal component $\mathbf{F}=\mathbf{U y}$ of the multivariate variable $\mathbf{y}$, where $\mathbf{U}=\left(\mathbf{u}_{1}, \ldots, \mathbf{u}_{p}\right)$ is the matrix of eigenvectors of the covariance matrix $\boldsymbol{\Sigma}$, corresponding to the eigenvalues $\lambda_{1}, \ldots, \lambda_{p}$. Srivastava measure is then written as

$$
\beta_{1 p}^{2}=\frac{1}{p} \sum_{i=1}^{p}\left\{\frac{E\left(F_{i}-\theta_{i}\right)^{3}}{\lambda_{i}^{\frac{3}{2}}}\right\}^{2},
$$

where $F_{i}=\mathbf{u}_{i}^{\top} \mathbf{y}$ and $\theta_{i}=\mathbf{u}_{i}^{\top} \boldsymbol{\mu}$. Thus this measure is based on central moments of third order $E\left[\mathbf{u}_{i}^{\top}(\mathbf{y}-\boldsymbol{\mu})\right]^{3}$. Based on the relationship between the central moments of third order $\left(\bar{M}_{3}(\mathbf{y})\right)$ and the non-central moments $\left(M_{3}(\mathbf{y})\right)$ and by using the relations for affine transformations of moments, we obtain the third central moments to be

$$
\begin{aligned}
E\left[\mathbf{u}_{i}^{\top}(\mathbf{y}-\boldsymbol{\mu})\right]^{3}= & \left(\mathbf{u}_{i}^{\top} \otimes \mathbf{u}_{i}^{\top}\right) \mathbf{M}_{3}(\mathbf{y}) \mathbf{u}_{i}-\left[\mathbf{u}_{i}^{\top} E\left(\mathbf{y} \mathbf{y}^{\top}\right) \mathbf{u}_{i}\right] \otimes\left[\mathbf{u}_{i}^{\top} E(\mathbf{y})\right] \\
& -\mathbf{u}_{i}^{\top} E(\mathbf{y}) \otimes\left[\mathbf{u}_{i}^{\top} E\left(\mathbf{y} \mathbf{y}^{\top}\right) \mathbf{u}_{i}\right]-\operatorname{vec}\left(\mathbf{u}_{i}^{\top} E\left(\mathbf{y} \mathbf{y}^{\top}\right) \mathbf{u}_{i}\right)(E(\mathbf{y}))^{\top} \mathbf{u}_{i} \\
& +2\left[\mathbf{u}_{i}^{\top} E(\mathbf{y})(E(\mathbf{y}))^{\top} \mathbf{u}_{i}\right] \otimes\left[\mathbf{u}_{i}^{\top} E(\mathbf{y})\right]
\end{aligned}
$$

where

$$
\begin{aligned}
& \mathbf{M}_{1}(\mathbf{y})=E(\mathbf{y})=\boldsymbol{\xi}+\sqrt{\frac{2}{\pi}} c_{1} \gamma, \\
& \mathbf{M}_{2}(\mathbf{y})=E\left(\mathbf{y} \mathbf{y}^{\top}\right)=c_{2} \boldsymbol{\Omega}+\boldsymbol{\xi} \boldsymbol{\xi}^{\top}+\sqrt{\frac{2}{\pi}} c_{1}\left(\xi \boldsymbol{\gamma}^{\top}+\gamma \boldsymbol{\xi}^{\top}\right),
\end{aligned}
$$

where $\gamma=\omega \delta$. 


\subsection{Móri-Rohatgi-Székely measure}

Móri et al. (1993) developed a vectorial skewness as:

$$
\begin{aligned}
s(\mathbf{y}) & =E\left(\|\mathbf{z}\|^{2} \mathbf{z}\right)=E\left\{\left(\mathbf{z}^{\top} \mathbf{z}\right) \mathbf{z}\right\}=\sum_{i=1}^{p} E\left(Z_{i}^{2} \mathbf{z}\right) \\
& =\left(\sum_{i=1}^{p} E\left(Z_{i}^{2} Z_{1}\right), \ldots, \sum_{i=1}^{p} E\left(Z_{i}^{2} Z_{p}\right)\right)^{\top},
\end{aligned}
$$

where $\mathbf{z}=\boldsymbol{\Sigma}^{-1 / 2}(\mathbf{y}-\boldsymbol{\mu})=\left(Z_{1}, \ldots, Z_{p}\right)^{\top}$. Note that this measure is location and scale invariant and $\mathbf{z}$ is still scale mixtures of multivariate skew-normal distributions when $\mathbf{y}$ is. Furthermore, replacing $\mathbf{u}_{i}$ by matrix $\mathbf{A}=\boldsymbol{\Sigma}^{-1 / 2}$ in (2.5), the equation still works since

$$
\begin{aligned}
& \mathbf{M}_{1}(\mathbf{A y})=E(\mathbf{A y})=\mathbf{A} E(\mathbf{y}) \\
& \mathbf{M}_{2}(\mathbf{A y})=E\left(\mathbf{A y}(\mathbf{A y})^{\top}\right)=\mathbf{A} E\left(\mathbf{y} \mathbf{y}^{\top}\right) \mathbf{A}^{\top}, \\
& \mathbf{M}_{3}(\mathbf{A y})=E\left((\mathbf{A y} \otimes \mathbf{A y})(\mathbf{A y})^{\top}\right)=E\left(\operatorname{vec}\left(\mathbf{A} \mathbf{y}(\mathbf{A y})^{\top}\right)(\mathbf{A y})^{\top}\right)=(\mathbf{A} \otimes \mathbf{A}) \mathbf{M}_{3}(\mathbf{y}) \mathbf{A}^{\top} .
\end{aligned}
$$

Therefore we can use the moments in (2.4) and (2.5) to obtain $s(\mathbf{y})$.

\subsection{Kollo measure}

Kollo (2008) noticed that $s(\mathbf{y})$ does not include all third order mixed moments. To include all mixed moments of the third order, he defined a skewness vector of $\mathbf{y}$ as:

$$
\begin{aligned}
b(\mathbf{y}) & =E\left(\sum_{i, j}^{p}\left(Z_{i} Z_{j}\right) \mathbf{z}\right) \\
& =\left(\sum_{i, j}^{p} E\left[\left(Z_{i} Z_{j}\right) Z_{1}\right], \ldots, \sum_{i, j}^{p} E\left[\left(Z_{i} Z_{j}\right) Z_{p}\right]\right)^{\top} .
\end{aligned}
$$

The required moments can be obtained from (2.4) and (2.5) by similar arguments in Section 2.6.

\subsection{Song measure of shape}

Song (2001) derived a general measure of the shape of a distribution, based on Rényi's entropy, as

$$
S(f)=\operatorname{var}[\log (f(\mathbf{y}))],
$$

where $f$ denotes the density function. For obtaining (2.6), we depend on the delta method as an approximation. That is,

$$
\operatorname{var}(G(\mathbf{y})) \approx\left[G^{\prime}(\boldsymbol{\mu})\right]^{\top} \operatorname{var}(\mathbf{y})\left[G^{\prime}(\boldsymbol{\mu})\right] .
$$

For scale mixtures of multivariate skew-normal distributions,

$$
\begin{aligned}
G(\mathbf{y}) & =\log \{f(\mathbf{y})\} \\
& =\log \left\{2 \int_{0}^{\infty} \phi_{p}(\mathbf{y} ; \boldsymbol{\xi}, W(\eta) \boldsymbol{\Omega}) \Phi\left\{W(\eta)^{-\frac{1}{2}} \boldsymbol{\alpha}^{\top} \boldsymbol{\omega}^{-1}(\mathbf{y}-\boldsymbol{\xi})\right\} d H(\eta)\right\} .
\end{aligned}
$$


The derivative is

$$
\begin{aligned}
G^{\prime}(\mathbf{y})= & \frac{1}{f(\mathbf{y})}\left\{\int _ { 0 } ^ { \infty } \left[-2 \phi_{p}(\mathbf{y} ; \boldsymbol{\xi}, W(\eta) \mathbf{\Omega}) \Phi\{\varsigma\} W(\eta)^{-1} \mathbf{\Omega}^{-1}(\mathbf{y}-\boldsymbol{\xi})\right.\right. \\
& \left.\left.+2 \phi_{p}(\mathbf{y} ; \boldsymbol{\xi}, W(\eta) \mathbf{\Omega}) \phi_{1}\{\varsigma\} W(\eta)^{-\frac{1}{2}} \omega^{-1} \boldsymbol{\alpha}\right] d H(\eta)\right\}
\end{aligned}
$$

where $\varsigma=W(\eta)^{-1 / 2} \boldsymbol{\alpha}^{\top} \boldsymbol{\omega}^{-1}(\mathbf{y}-\boldsymbol{\xi})$. From the above facts

$$
S(f) \approx\left[G^{\prime}(\boldsymbol{\mu})\right]^{\top} \operatorname{var}(\mathbf{y})\left[G^{\prime}(\boldsymbol{\mu})\right],
$$

where $\boldsymbol{\mu}=E(\mathbf{y})=\boldsymbol{\xi}+\sqrt{2 / \pi} c_{1} \boldsymbol{\gamma}$ and $\operatorname{var}(\mathbf{y})=c_{2} \boldsymbol{\Omega}-(2 / \pi) c_{1}^{2} \boldsymbol{\gamma} \boldsymbol{\gamma}^{\top}$.

For skew- $t$ distribution, we obtain a closed form of this measure. That is,

$$
\begin{aligned}
G(\mathbf{y}) & =\log \{f(\mathbf{y})\} \\
& \propto-\frac{v+p}{2} \log \left(1+\frac{Q_{\mathbf{y}}}{v}\right)+\log T_{1}\left\{\boldsymbol{\alpha}^{\top} \boldsymbol{\omega}^{-1}(\mathbf{y}-\boldsymbol{\xi})\left(\frac{v+p}{Q_{\mathbf{y}}+v}\right)^{\frac{1}{2}} ; v+p\right\} .
\end{aligned}
$$

Its derivative is

$$
G^{\prime}(\mathbf{y})=-\frac{(v+p) \boldsymbol{\Omega}^{-1}(\mathbf{y}-\boldsymbol{\xi})}{v\left(1+Q_{\mathbf{y}} / v\right)}+\left\{\left(\frac{v+p}{Q_{\mathbf{y}}+v}\right)^{\frac{1}{2}} \omega^{-1} \boldsymbol{\alpha}-\frac{\kappa}{Q_{\mathbf{y}}+v} \mathbf{\Omega}^{-1}(\mathbf{y}-\boldsymbol{\xi})\right\} \frac{t_{1}(\kappa ; v+p)}{T_{1}(\kappa ; v+p)},
$$

where $Q_{\mathbf{y}}=(\mathbf{y}-\boldsymbol{\xi})^{\top} \mathbf{\Omega}^{-1}(\mathbf{y}-\boldsymbol{\xi}), \kappa=\boldsymbol{\alpha}^{\top} \boldsymbol{\omega}^{-1}(\mathbf{y}-\boldsymbol{\xi})\left\{(v+p) /\left(Q_{\mathbf{y}}+v\right)\right\}^{1 / 2}$ and $t_{1}, T_{1}$ are the density function and distribution function of skew- $t$ distribution respectively. From the above facts

$$
S(f) \approx\left[G^{\prime}(\boldsymbol{\mu})\right]^{\top} \operatorname{var}(\mathbf{y})\left[G^{\prime}(\boldsymbol{\mu})\right],
$$

where $E(\mathbf{y})=\boldsymbol{\mu}=\boldsymbol{\xi}+\boldsymbol{\omega} \boldsymbol{\mu}_{\mathbf{z}}, \boldsymbol{\mu}_{\mathbf{z}}=b_{v} \boldsymbol{\delta}, b_{v}=\{\sqrt{v} \Gamma((v-1) / 2)\} /\{\sqrt{\pi} \Gamma(v / 2)\}, \boldsymbol{\delta}=\{\overline{\mathbf{\Omega}} \boldsymbol{\alpha}\} /\left\{\left(1+\boldsymbol{\alpha}^{\top} \overline{\mathbf{\Omega}} \boldsymbol{\alpha}\right)^{1 / 2}\right\}$, and $\operatorname{var}(\mathbf{y})=\{v /(v-2)\} \mathbf{\Omega}-\boldsymbol{\omega} \boldsymbol{\mu}_{\mathbf{z}} \boldsymbol{\mu}_{\mathbf{z}}^{\top} \boldsymbol{\omega}$.

\section{Comparison of measures}

To compare the above multivariate measures of skewness for the scale mixtures of skew-normal distributions, we did a simulation study for two special cases, skew- $t$ and skew-normal distributions since they are the most important family of distributions within the class.

In the simulation study, we use $\boldsymbol{\xi}=\mathbf{0}_{p}, \boldsymbol{v}=5$, the parameters $\boldsymbol{\Omega}$ and $\boldsymbol{\alpha}$ are given in tables. Tables 3, 4 and 5, 6 present the values of multivariate measures of skewness for different choices of parameters of the bivariate skew- $t$ distribution and bivariate skew-normal distribution along with corresponding contour plots. In each contour plot, the black straight line illustrates the direction of $\mathbf{t}$ in the Balakrishnan-Brito-Quiroz measure and the blue one shows the direction of $\delta$ in the Isogai measure.

For the skew- $t$ distribution, Mardia's index appears to be always larger than the MalkovichAfifi measure. $Q^{*}$ values in Balakrishnan-Brito-Quiroz measure present completely different ranking among all considered cases: the values are much greater relatively. In cases 3 and 4, Mardia, Malkovich-Afifi, and Song individually behave the same. Compared to the measures of skew-normal distribution, those of skew- $t$ distribution present a larger scale of skewness and the skewness seems 
Table 3: Skewness measure for some bivariate skew- $t$ distributions (1)

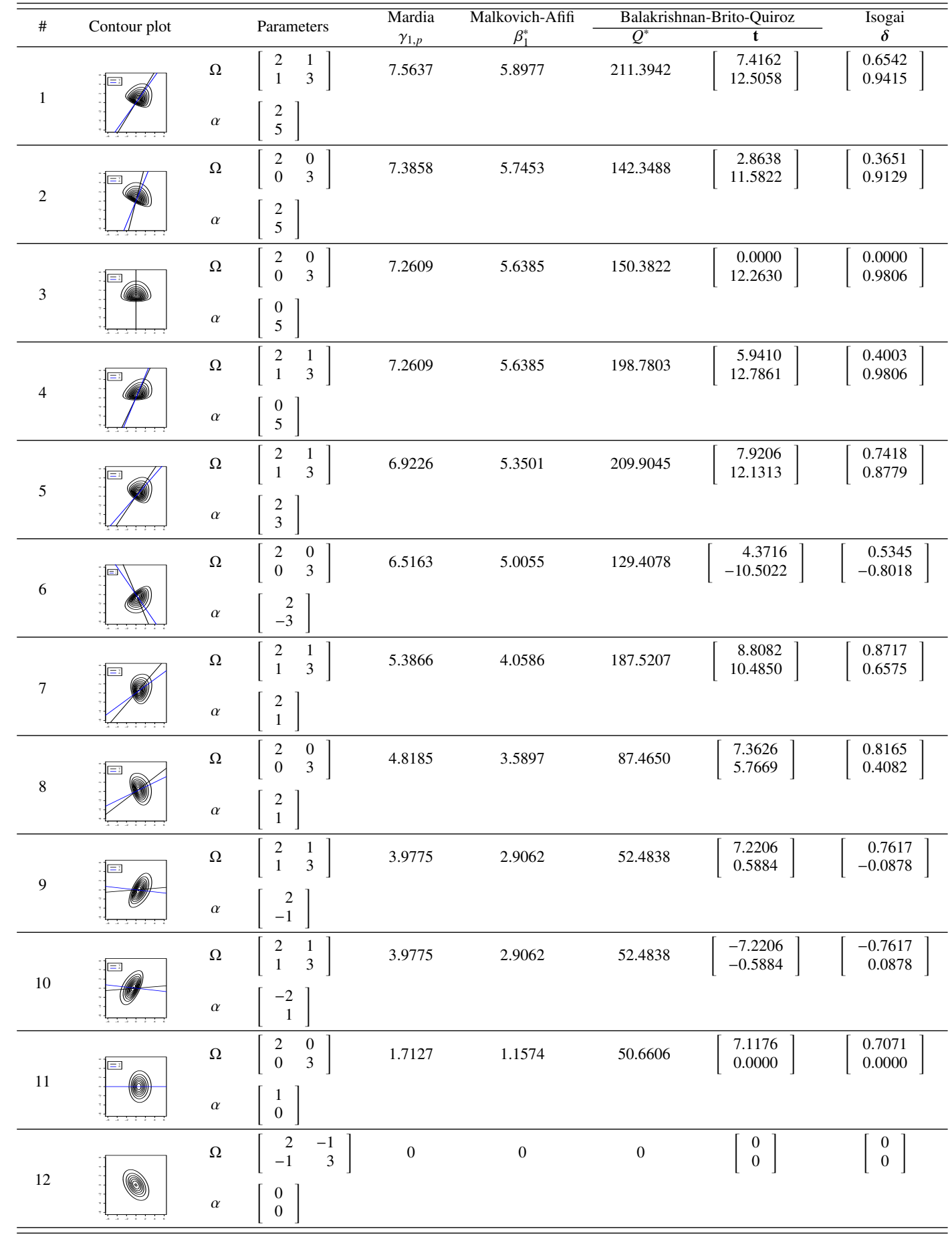


Table 4: Skewness measure for some bivariate skew- $t$ distributions (2)

\begin{tabular}{|c|c|c|c|c|c|c|c|}
\hline \# & Contour plot & & Parameters & $\begin{array}{c}\text { Srivastava } \\
\beta_{1 p}^{2} \\
\end{array}$ & $\begin{array}{c}\text { Mori-Rohatgi-Szekely } \\
s(\mathbf{y}) \\
\end{array}$ & $\begin{array}{c}\text { Kollo } \\
b(\mathbf{y})\end{array}$ & $\begin{array}{l}\text { Song } \\
S(f)\end{array}$ \\
\hline \multirow{2}{*}{1} & & $\Omega$ & $\left.\begin{array}{ll}2 & 1 \\
1 & 3\end{array}\right]$ & 2.7108 & {$\left[\begin{array}{l}1.3840 \\
2.6114\end{array}\right]$} & $\begin{array}{l}2.6437 \\
3.7247\end{array}$ & 0.9804 \\
\hline & & $\alpha$ & $\left.\begin{array}{l}2 \\
5\end{array}\right]$ & & & & \\
\hline \multirow{2}{*}{2} & & $\Omega$ & $\begin{array}{ll}2 & 0 \\
0 & 3\end{array}$ & 2.6218 & $\begin{array}{l}1.1193 \\
2.6968\end{array}$ & $\begin{array}{l}2.3100 \\
3.6393\end{array}$ & 0.9810 \\
\hline & & $\alpha$ & $\left.\begin{array}{l}2 \\
5\end{array}\right]$ & & & & \\
\hline \multirow{2}{*}{3} & & $\Omega$ & $\begin{array}{ll}2 & 0 \\
0 & 3\end{array}$ & 2.8193 & $\begin{array}{l}0.0000 \\
2.8946\end{array}$ & $\begin{array}{l}1.0400 \\
2.8946\end{array}$ & 0.9808 \\
\hline & & $\alpha$ & $\left.\begin{array}{l}0 \\
5\end{array}\right]$ & & & & \\
\hline \multirow{2}{*}{4} & & $\Omega$ & $\begin{array}{ll}2 & 1 \\
1 & 3\end{array}$ & 1.9386 & $\left.\begin{array}{l}0.4590 \\
2.8579\end{array}\right]$ & $\begin{array}{l}1.5263 \\
3.2747\end{array}$ & 0.9808 \\
\hline & & $\alpha$ & $\left.\begin{array}{l}0 \\
5\end{array}\right]$ & & & & \\
\hline \multirow{2}{*}{5} & & $\Omega$ & $\begin{array}{ll}2 & 1 \\
1 & 3\end{array}$ & 2.4052 & $\begin{array}{l}1.6916 \\
2.2625\end{array}$ & $\begin{array}{l}2.9580 \\
3.4727\end{array}$ & 0.9767 \\
\hline & & $\alpha$ & $\left.\begin{array}{l}2 \\
3\end{array}\right]$ & & & & \\
\hline \multirow{2}{*}{6} & 国 & $\Omega$ & $\begin{array}{ll}2 & 0 \\
0 & 3\end{array}$ & 2.2678 & $\begin{array}{r}1.5561 \\
-2.2542\end{array}$ & $\begin{array}{r}0.3414 \\
-1.1208\end{array}$ & 0.9638 \\
\hline & & $\alpha$ & $\left.\begin{array}{r}2 \\
-3\end{array}\right]$ & & & & \\
\hline \multirow{2}{*}{7} & 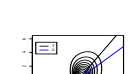 & $\Omega$ & $\begin{array}{ll}2 & 1 \\
1 & 3\end{array}$ & 1.4678 & $\begin{array}{l}2.0980 \\
1.3319\end{array}$ & $\begin{array}{l}3.0632 \\
2.4189\end{array}$ & 0.8782 \\
\hline & & $\alpha$ & $\left.\begin{array}{l}2 \\
1\end{array}\right]$ & & & & \\
\hline \multirow{2}{*}{8} & $\Xi$ & $\Omega$ & $\begin{array}{ll}2 & 0 \\
0 & 3\end{array}$ & 1.7371 & $\begin{array}{l}2.1117 \\
1.0247\end{array}$ & $\begin{array}{l}2.8863 \\
2.0231\end{array}$ & 0.8092 \\
\hline & & $\alpha$ & $\left.\begin{array}{l}2 \\
1\end{array}\right]$ & & & & \\
\hline \multirow{2}{*}{9} & 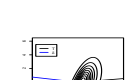 & $\Omega$ & $\begin{array}{ll}2 & 1 \\
1 & 3\end{array}$ & 1.4178 & $\left.\begin{array}{r}2.0295 \\
-0.6376\end{array}\right]$ & $\begin{array}{l}1.5377 \\
0.2435\end{array}$ & 0.6831 \\
\hline & & $\alpha$ & & & & & \\
\hline 10 & $\Phi$ & $\Omega$ & $\begin{array}{ll}2 & 1 \\
1 & 3\end{array}$ & 1.4178 & $\begin{array}{r}-2.0295 \\
0.6376\end{array}$ & $\begin{array}{l}-1.5377 \\
-0.2435\end{array}$ & 0.6831 \\
\hline 10 & & $\alpha$ & $\left.\begin{array}{r}-2 \\
1\end{array}\right]$ & & & & \\
\hline & 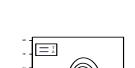 & $\Omega$ & $\begin{array}{ll}2 & 0 \\
0 & 3\end{array}$ & 0.5787 & $\begin{array}{l}1.3800 \\
0.0000\end{array}$ & $\begin{array}{l}1.3800 \\
0.6085\end{array}$ & 0.2809 \\
\hline 11 & ד: & $\alpha$ & $\left.\begin{array}{l}1 \\
0\end{array}\right]$ & & & & \\
\hline & & $\Omega$ & $\begin{array}{rr}2 & -1 \\
-1 & 3\end{array}$ & 0 & $\left.\begin{array}{l}0 \\
0\end{array}\right]$ & $\left.\begin{array}{l}0 \\
0\end{array}\right]$ & 0 \\
\hline 12 & $+2+1$ & $\alpha$ & $\left.\begin{array}{l}0 \\
0\end{array}\right]$ & & & & \\
\hline
\end{tabular}


Table 5: Skewness measure for some bivariate skew-normal distributions (1)

\begin{tabular}{|c|c|c|c|c|c|c|c|c|}
\hline \multirow{2}{*}{$\#$} & \multirow{2}{*}{ Contour plot } & & \multirow{2}{*}{ Parameters } & \multirow{2}{*}{$\begin{array}{c}\text { Mardia } \\
\gamma_{1, p} \\
\end{array}$} & \multirow{2}{*}{$\begin{array}{c}\text { Malkovich-Afifi } \\
\beta_{1}^{*}\end{array}$} & \multicolumn{2}{|c|}{ Balakrishnan-Brito-Quiroz } & \multirow{2}{*}{$\begin{array}{c}\text { Isogai } \\
\delta\end{array}$} \\
\hline & & & & & & $Q^{*}$ & $\mathbf{t}$ & \\
\hline \multirow{2}{*}{1} & & $\Omega$ & $\left.\begin{array}{ll}2 & 1 \\
1 & 3\end{array}\right]$ & 0.7995 & 0.7995 & 23.9081 & $\begin{array}{l}2.4941 \\
4.2057\end{array}$ & $\begin{array}{l}0.6542 \\
0.9415\end{array}$ \\
\hline & & $\alpha$ & $\left.\begin{array}{l}2 \\
5\end{array}\right]$ & & & & & \\
\hline \multirow{2}{*}{2} & & $\Omega$ & $\begin{array}{ll}2 & 0 \\
0 & 3\end{array}$ & 0.7547 & 0.7547 & 16.0993 & $\begin{array}{l}0.9631 \\
3.8951\end{array}$ & $\begin{array}{l}0.3651 \\
0.9129\end{array}$ \\
\hline & & $\alpha$ & $\left.\begin{array}{l}2 \\
5\end{array}\right]$ & & & & & \\
\hline \multirow{2}{*}{3} & & $\Omega$ & $\begin{array}{ll}2 & 0 \\
0 & 3\end{array}$ & 0.7241 & 0.7241 & 17.0078 & $\begin{array}{l}0.0000 \\
4.1241\end{array}$ & $\begin{array}{l}0.0000 \\
0.9806\end{array}$ \\
\hline & & $\alpha$ & \begin{tabular}{l|l}
0 & \\
5 &
\end{tabular} & & & & & \\
\hline \multirow{2}{*}{4} & & $\Omega$ & $\begin{array}{ll}2 & 1 \\
1 & 3\end{array}$ & 0.7241 & 0.7241 & 22.4815 & $\begin{array}{l}1.9980 \\
4.2999\end{array}$ & $\begin{array}{l}0.4003 \\
0.9806\end{array}$ \\
\hline & & $\alpha$ & $\begin{array}{l}0 \\
5\end{array}$ & & & & & \\
\hline \multirow{2}{*}{5} & & $\Omega$ & $\begin{array}{ll}2 & 1 \\
1 & 3\end{array}$ & 0.6450 & 0.6450 & 23.7396 & $\begin{array}{l}2.6637 \\
4.0798\end{array}$ & $\begin{array}{l}0.7418 \\
0.8779\end{array}$ \\
\hline & & $\alpha$ & $\left.\begin{array}{l}2 \\
3\end{array}\right]$ & & & & & \\
\hline \multirow{2}{*}{6} & & $\Omega$ & $\begin{array}{ll}2 & 0 \\
0 & 3\end{array}$ & 0.5568 & 0.5568 & 14.6357 & $\begin{array}{r}1.4702 \\
-3.5319\end{array}$ & $\begin{array}{r}0.5345 \\
-0.8018\end{array}$ \\
\hline & & $\alpha$ & {$\left[\begin{array}{r}2 \\
-3\end{array}\right]$} & & & & & \\
\hline \multirow{2}{*}{7} & & $\Omega$ & $\begin{array}{ll}2 & 1 \\
1 & 3\end{array}$ & 0.3497 & 0.3497 & 21.2081 & $\begin{array}{l}2.9622 \\
3.5261\end{array}$ & $\begin{array}{l}0.8717 \\
0.6575\end{array}$ \\
\hline & & $\alpha$ & $\left.\begin{array}{l}2 \\
1\end{array}\right]$ & & & & & \\
\hline \multirow{2}{*}{8} & & $\Omega$ & {$\left[\begin{array}{ll}2 & 0 \\
0 & 3\end{array}\right.$} & 0.2658 & 0.2658 & 9.8921 & $\begin{array}{l}2.4760 \\
1.9394\end{array}$ & $\begin{array}{l}0.8165 \\
0.4082\end{array}$ \\
\hline & & $\alpha$ & $\left.\begin{array}{l}2 \\
1\end{array}\right]$ & & & & & \\
\hline \multirow{2}{*}{9} & $\Phi$ & $\Omega$ & $\begin{array}{ll}2 & 1 \\
1 & 3\end{array}$ & 0.1650 & 0.1650 & 5.9358 & $\begin{array}{l}2.4283 \\
0.1979\end{array}$ & $\begin{array}{r}0.7617 \\
-0.0878\end{array}$ \\
\hline & & $\alpha$ & $\left.\begin{array}{r}2 \\
-1\end{array}\right]$ & & & & & \\
\hline \multirow{2}{*}{10} & 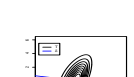 & $\Omega$ & {$\left[\begin{array}{ll}2 & 1 \\
1 & 3\end{array}\right.$} & 0.1650 & 0.1650 & 5.9358 & $\begin{array}{l}-2.4283 \\
-0.1979\end{array}$ & $\begin{array}{r}-0.7617 \\
0.0878\end{array}$ \\
\hline & & $\alpha$ & $\left.\begin{array}{r}-2 \\
1\end{array}\right]$ & & & & & \\
\hline \multirow{2}{*}{11} & 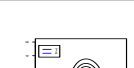 & $\Omega$ & $\begin{array}{ll}2 & 0 \\
0 & 3\end{array}$ & 0.0188 & 0.0188 & 5.7296 & $\begin{array}{l}2.3937 \\
0.0000\end{array}$ & $\begin{array}{l}0.7071 \\
0.0000\end{array}$ \\
\hline & & $\alpha$ & $\begin{array}{l}1 \\
0\end{array}$ & & & & & \\
\hline \multirow{2}{*}{12} & & $\Omega$ & $\begin{array}{rr}2 & -1 \\
-1 & 3\end{array}$ & 0 & 0 & 0 & $\left.\begin{array}{l}0 \\
0\end{array}\right]$ & $\left.\begin{array}{l}0 \\
0\end{array}\right]$ \\
\hline & & $\alpha$ & $\left.\begin{array}{l}0 \\
0\end{array}\right]$ & & & & & \\
\hline
\end{tabular}


Table 6: Skewness measure for some bivariate skew-normal distributions (2)

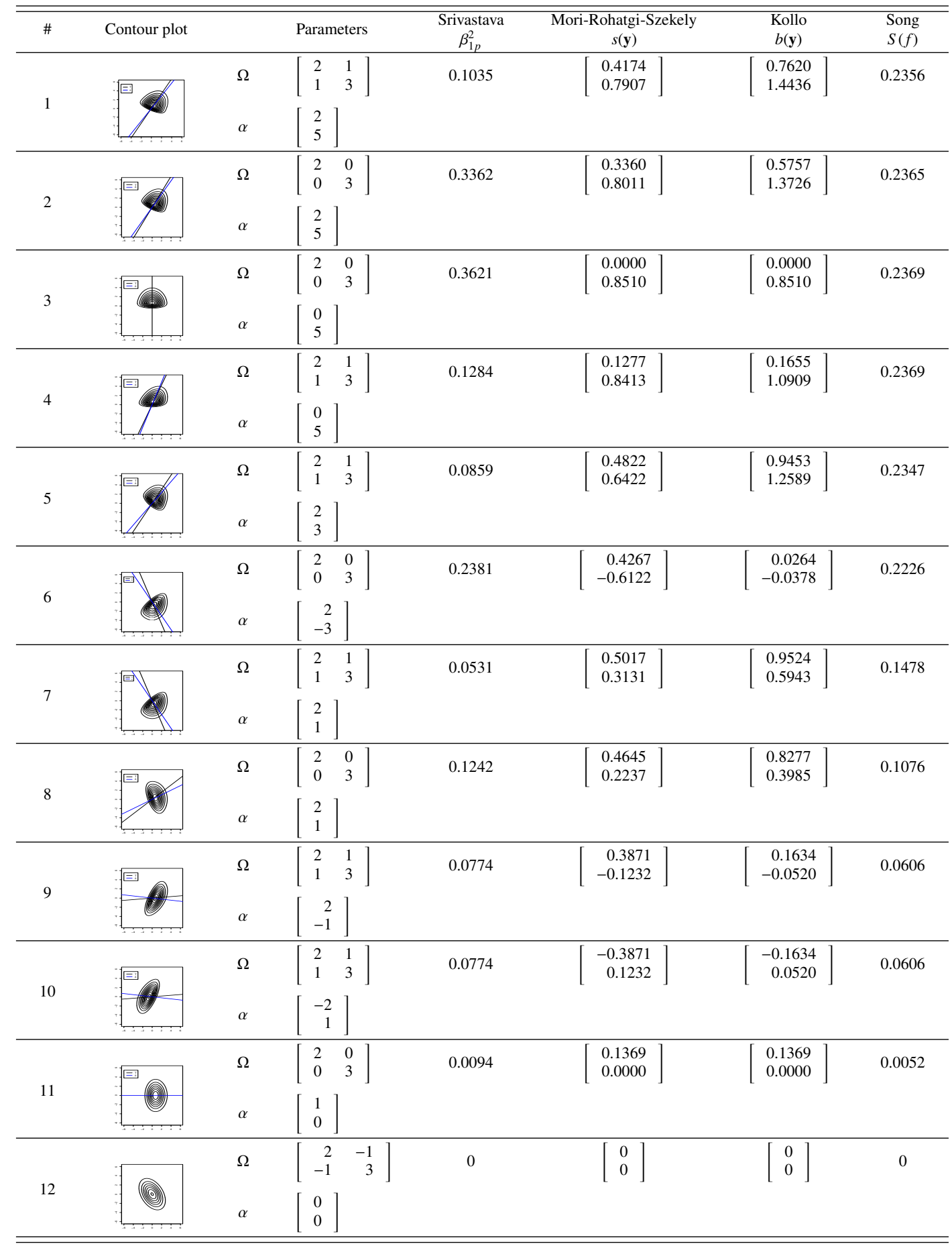


Table 7: Skewness measure for some trivariate skew- $t$ distributions (1)

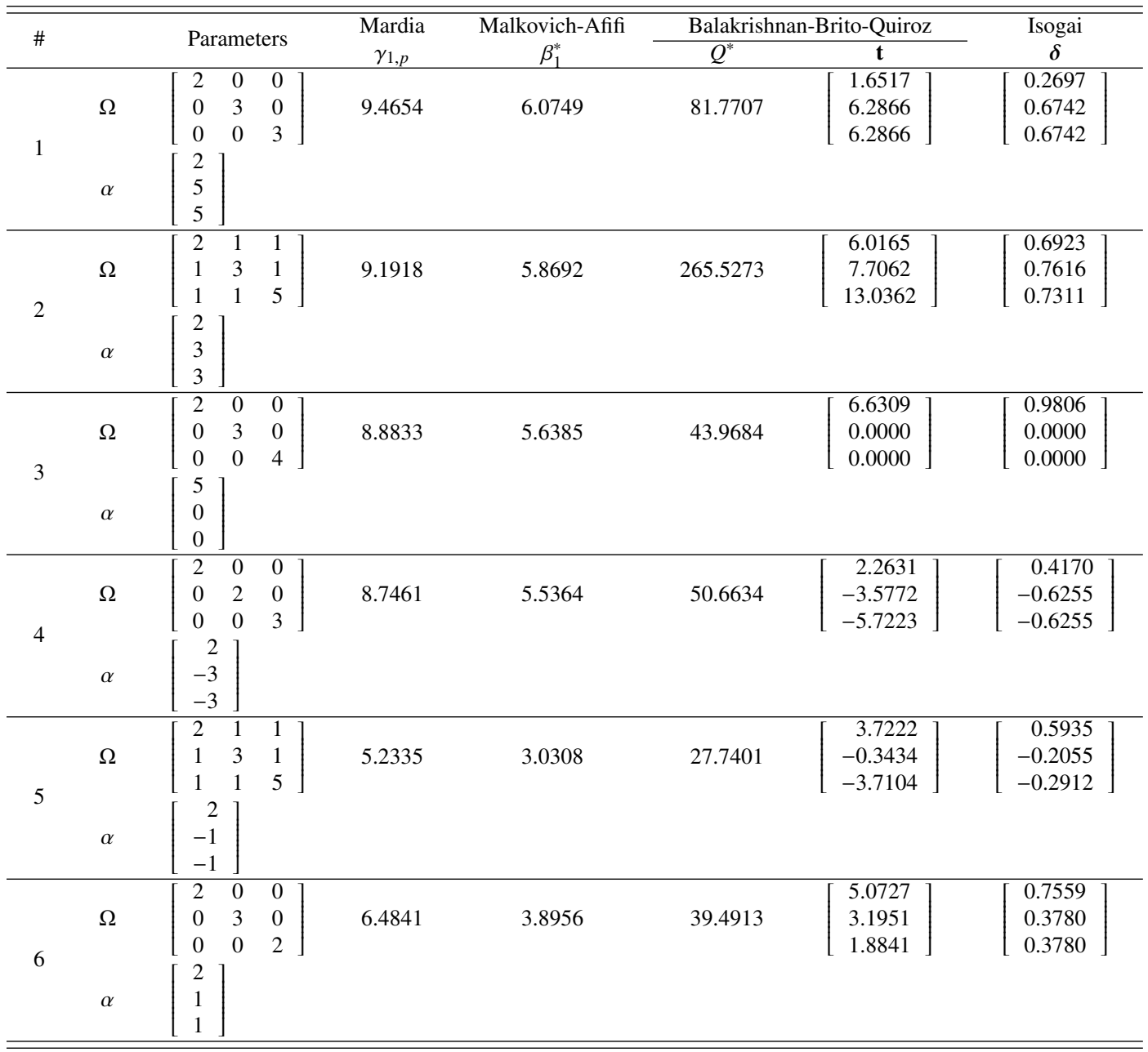

more active. The Mardia indexes and $Q^{*}$ values in Balakrishnan-Brito-Quiroz measure of skew- $t$ distribution are almost ten times than those of skew-normal distribution. However, as the degree of freedom $v$ increases, all measures converge to those of skew-normal distributions.

The difference between $S_{C}$ vectors of the Isogai measure in skew- $t$ and skew-normal distributions is the coefficients of $\boldsymbol{\delta}$. The two directions are therefore the same in the corresponding contour plots of the two distributions. The Srivastava measure in skew- $t$ distribution is over ten times greater than that of the skew-normal distribution. For the Song measure, in both skew- $t$ and skew-normal distributions, it presents a slightly different ranking among the cases 1-4. And in the same cases, the skew- $t$ distributions have very similar values around 0.98 which is greater than the values of skew-normal distribution varying around 0.23 .

We also found that in the skew- $t$ distribution, the Mardia index and Malkovich-Afifi measure behave differently, while they are same in skew-normal distribution. The vectorial measures yield very similar results in terms of skewness direction at both distributions. Finally we mention again 
Table 8: Skewness measure for some trivariate skew- $t$ distributions (2)

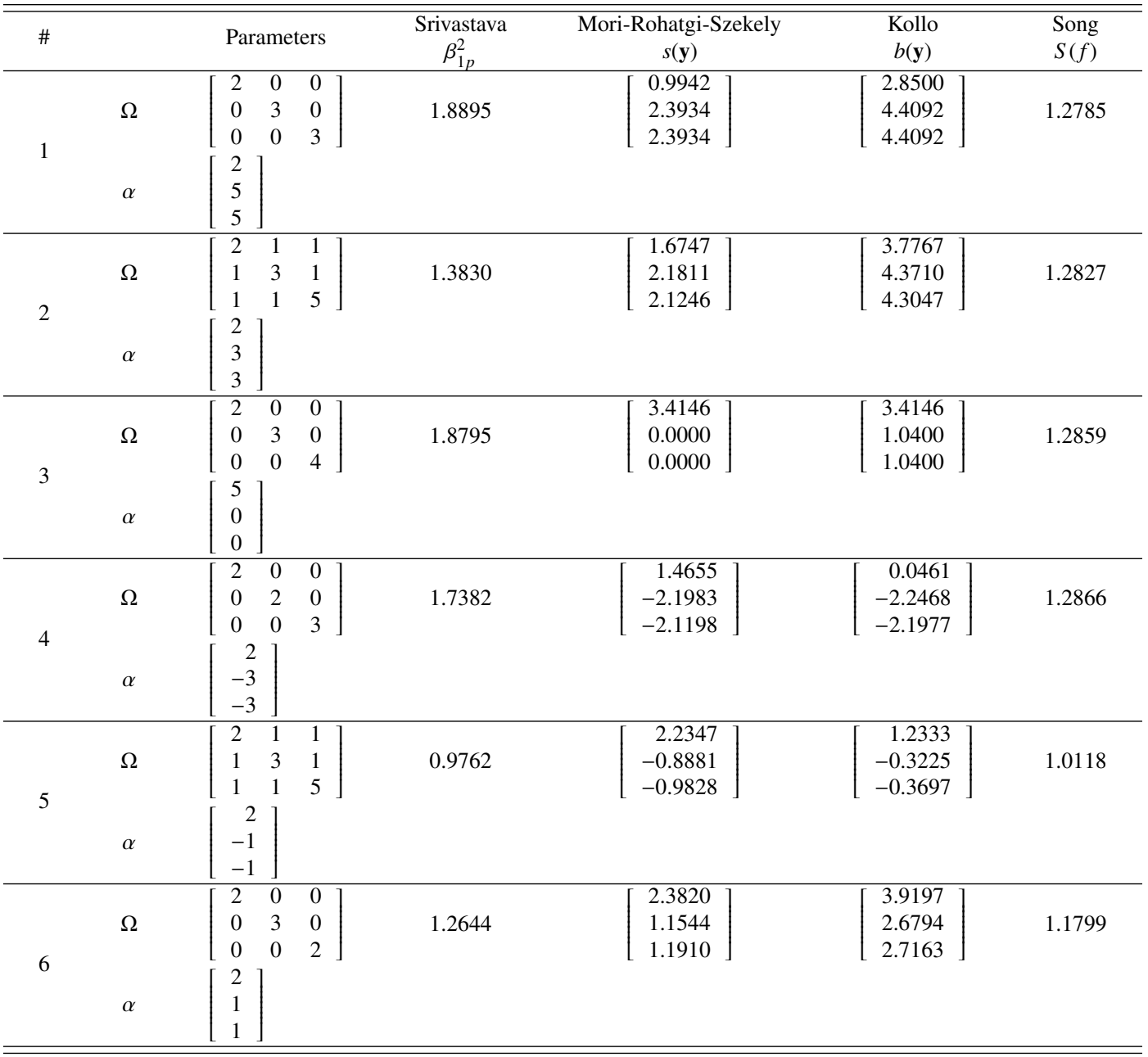

that, as the degree of freedom $v$ increases, all measures of skew- $t$ distributions converge to those of skew-normal distributions.

With the different choices of the parameters, Tables 7, 8 and 9, 10 illustrate the values of multivariate measures of skewness for trivariate skew- $t$ distribution and trivariate skew-normal distribution. For the trivariate skew- $t$ distribution, among all multivariate skewness measures, the difference of both ranking of skewness and scale of measures are similar to bivariate skew- $t$ distribution. The difference between trivariate skew- $t$ distribution and trivariate skew-normal distribution for each measure also agree with those in the bivariate conditions.

\section{Performance of the measures and comparisons}

Previous sections indicate that some measures are vectors that make it difficult for a direct comparison; therefore, it is better to take all measures in the same scale when comparing them. In order to attain a set of comparable indices, the sample version is considered for each of the skewness measures that 
Table 9: Skewness measure for some trivariate skew-normal distributions (1)

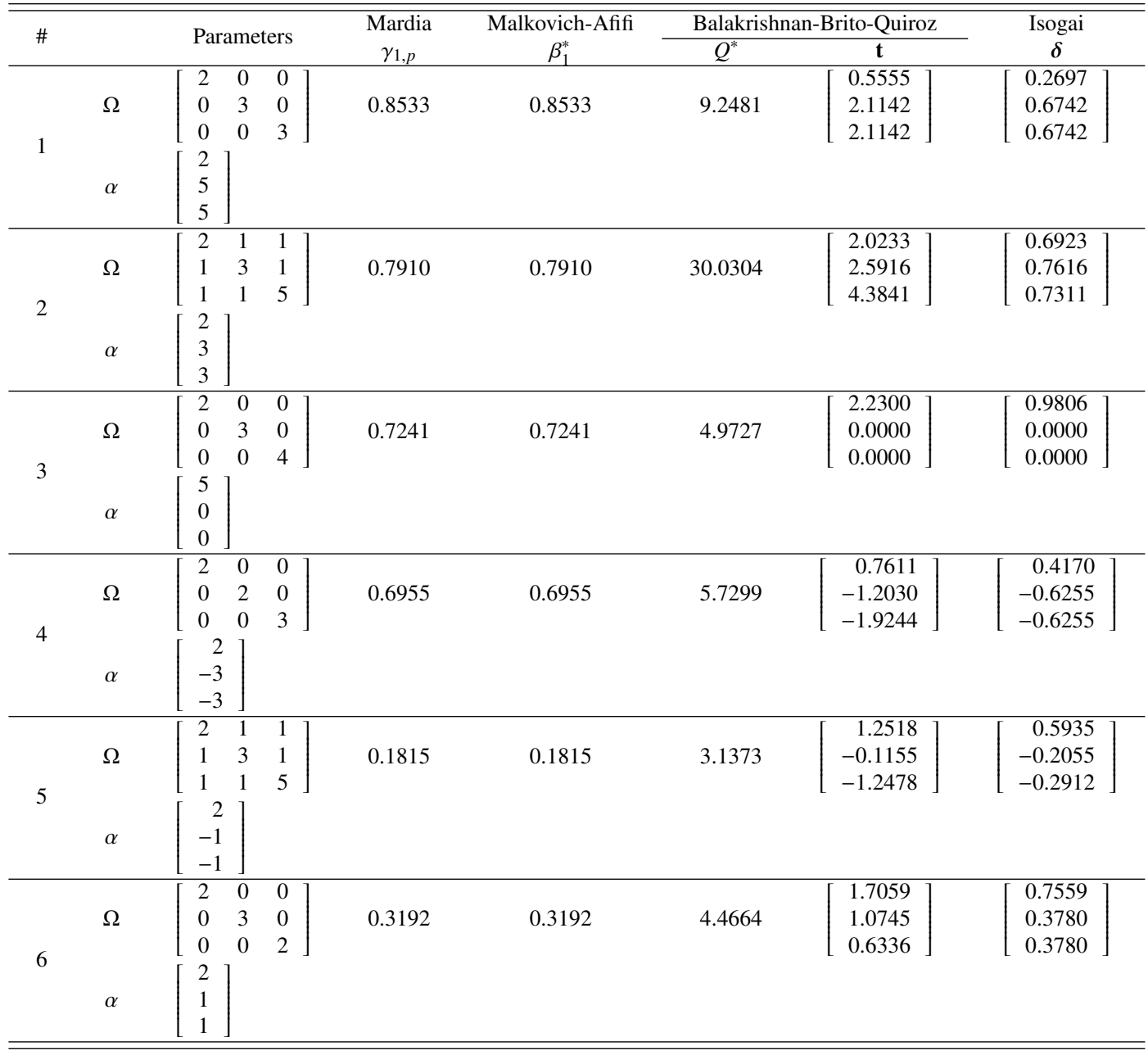

are taken as test statistics for the hypothesis of $t$ distribution against skew- $t$ distribution.

According to Balakrishnan and Scarpa (2012), $p$-value and power of that test are probabilities and can quantify the ability of each multivariate skewness measure to identify the specific asymmetry present in the skew- $t$ distribution within the same scale. In the simulation process, the sensitivity index ( $p$-value) for all multivariate skewness measures is attained by enumerating the number of samples from multivariate $t$ distribution having each skewness index not exceeding the theoretical value obtained for skew- $t$ distribution. And the specificity index (power of the test) is provided by sampling from skew- $t$ distribution and enumerating the number of samples which have each skewness measure not exceeding the expected theoretical value. While for the Songs measure, two values are obtained by considering in a reverse way, that is, the corresponding index exceeding the theoretical value.

In each sample version, all skewness measures are obtained by replacing $\boldsymbol{\xi}, v, \boldsymbol{\Omega}$ and $\boldsymbol{\alpha}$ (given in Tables 3, 4 and 5,6) with the maximum likelihood estimates of these quantities. In sensitivity 
Table 10: Skewness measure for some trivariate skew-normal distributions (2)

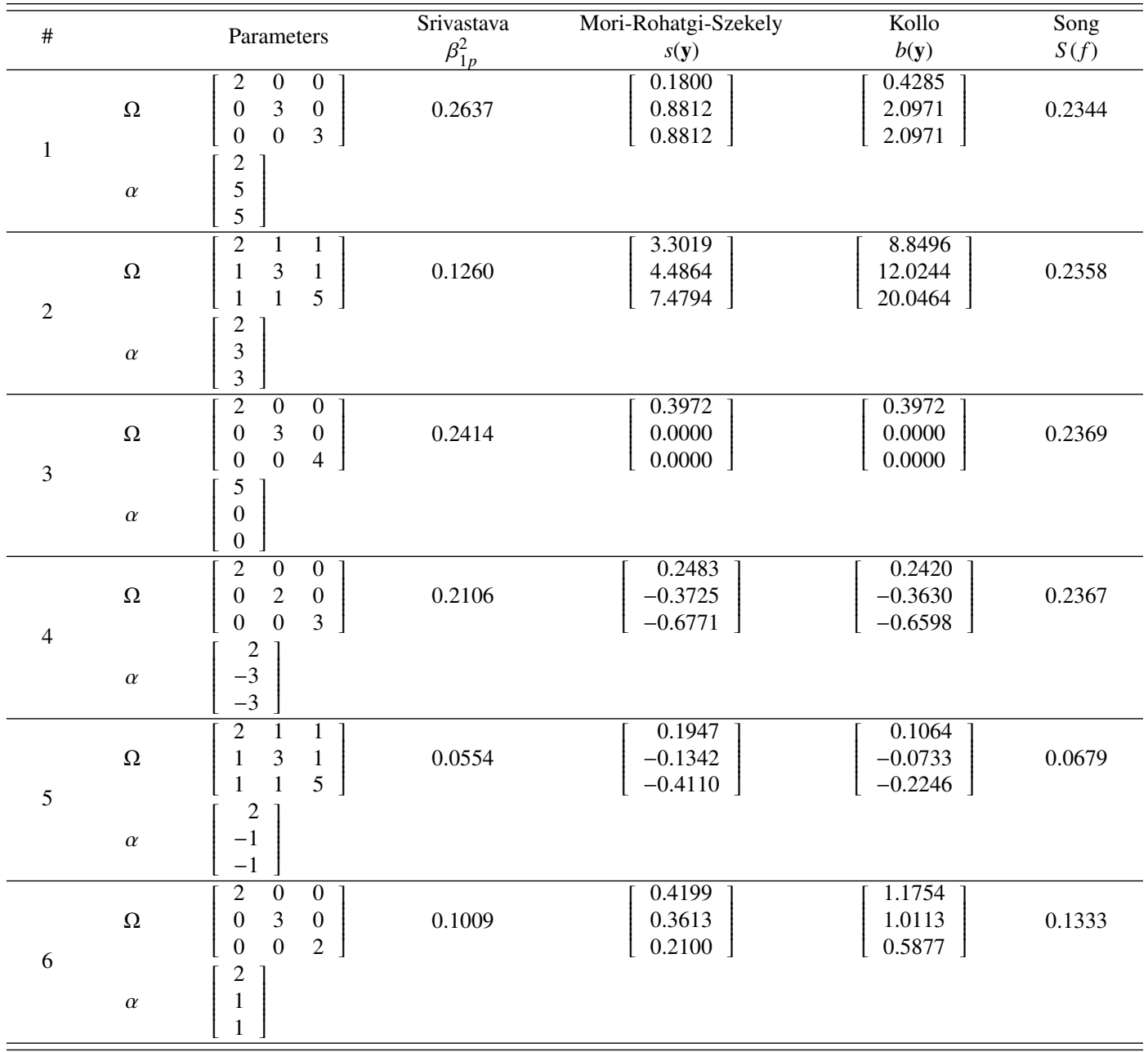

analysis, 1,000 samples with size 100 from the bivariate and trivariate $t$ distribution are simulated. Besides, specificity is attained by sampling 1,000 samples with size 100 from the bivariate and trivariate skew- $t$ distribution. We obtain a single test statistic, so the sum and maximum of the elements in each vectorial measure are provided for vectorial measures such as Balakrishnan-Brito-Quiroz, Isogai, Mori-Rohatgi-Szekely and Kollo.

The comparison among all measures can be performed directly based on the simulation results in Tables 11-14. In many cases, specificity is high for all measures, while sensitivity displays more variability. Balakrishnan-Brito-Quiroz measure seems to be the best choice in all considered cases.

\section{Application}

An application is reported for the data set consisting of 71 total glycerol and magnesium contents in Grignolino wine. The data are available by the R package "sn". Refer to Azzalini and Capitanio 
Table 11: $p$-value and power for test for bivariate $t$ distribution against skew- $t$ distribution (1)

\begin{tabular}{|c|c|c|c|c|c|c|c|c|c|c|c|}
\hline \multirow{2}{*}{$\#$} & \multirow{2}{*}{ Contour plot } & \multirow{2}{*}{\multicolumn{3}{|c|}{ Parameters }} & \multirow{3}{*}{$\begin{array}{c}\begin{array}{c}\text { Mardia } \\
\gamma_{1, p}\end{array} \\
0.328\end{array}$} & \multirow{3}{*}{$\begin{array}{c}\begin{array}{c}\text { Malkovich-Afifi } \\
\beta_{1}^{*}\end{array} \\
0.290\end{array}$} & \multicolumn{3}{|c|}{ Balakrishnan-Brito-Quiroz } & \multicolumn{2}{|c|}{ Isogai } \\
\hline & & & & & & & $Q^{*}$ & $t_{\text {sup }}$ & $t_{\text {sum }}$ & $\delta_{\text {sup }}$ & $\delta_{\text {sum }}$ \\
\hline \multirow{2}{*}{1} & & $\Omega$ & $\begin{array}{ll}2 & 1 \\
1 & 3\end{array}$ & $p$-value & & & 0.910 & 0.951 & 0.962 & 0.471 & 0.402 \\
\hline & & $\alpha$ & $\left.\begin{array}{l}2 \\
5\end{array}\right]$ & Power & 0.411 & 0.413 & 0.530 & 0.575 & 0.565 & 0.615 & 0.645 \\
\hline \multirow{2}{*}{2} & & $\Omega$ & $\begin{array}{ll}2 & 0 \\
0 & 3\end{array}$ & $p$-value & 0.694 & 0.682 & 0.864 & 0.927 & 0.944 & 0.750 & 0.932 \\
\hline & & $\alpha$ & $\left.\begin{array}{l}2 \\
5\end{array}\right]$ & Power & 0.262 & 0.264 & 0.539 & 0.564 & 0.568 & 0.190 & 0.774 \\
\hline \multirow{2}{*}{3} & & $\Omega$ & $\begin{array}{ll}2 & 0 \\
0 & 3\end{array}$ & $p$-value & 0.326 & 0.305 & 0.864 & 0.931 & 0.941 & 0.907 & 0.739 \\
\hline & & $\alpha$ & $\left.\begin{array}{l}0 \\
5\end{array}\right]$ & Power & 0.592 & 0.591 & 0.534 & 0.560 & 0.566 & 0.850 & 0.331 \\
\hline \multirow{2}{*}{4} & & $\Omega$ & $\begin{array}{ll}2 & 1 \\
1 & 3\end{array}$ & $p$-value & 0.213 & 0.309 & 0.873 & 0.934 & 0.940 & 0.259 & 0.355 \\
\hline & & $\alpha$ & $\left.\begin{array}{l}0 \\
5\end{array}\right]$ & Power & 0.667 & 0.666 & 0.548 & 0.577 & 0.582 & 0.697 & 0.982 \\
\hline \multirow{2}{*}{5} & & $\Omega$ & $\begin{array}{ll}2 & 1 \\
1 & 3\end{array}$ & $p$-value & 0.236 & 0.212 & 0.891 & 0.932 & 0.941 & 0.847 & 0.753 \\
\hline & & $\alpha$ & $\left.\begin{array}{l}2 \\
3\end{array}\right]$ & Power & 0.521 & 0.519 & 0.564 & 0.579 & 0.589 & 0.192 & 0.085 \\
\hline \multirow{2}{*}{6} & & $\Omega$ & $\begin{array}{ll}2 & 0 \\
0 & 3\end{array}$ & $p$-value & 0.499 & 0.533 & 0.872 & 0.766 & 0.149 & 0.514 & 0.743 \\
\hline & & $\alpha$ & $\left.\begin{array}{r}2 \\
-3\end{array}\right]$ & Power & 0.614 & 0.614 & 0.545 & 0.568 & 0.437 & 0.712 & 0.667 \\
\hline \multirow{2}{*}{7} & & $\Omega$ & $\begin{array}{ll}2 & 1 \\
1 & 3\end{array}$ & $p$-value & 0.490 & 0.469 & 0.877 & 0.926 & 0.958 & 0.088 & 0.078 \\
\hline & & $\alpha$ & $\left.\begin{array}{l}2 \\
1\end{array}\right]$ & Power & 0.752 & 0.751 & 0.592 & 0.579 & 0.615 & 0.390 & 0.849 \\
\hline \multirow{2}{*}{8} & & $\Omega$ & $\begin{array}{ll}2 & 0 \\
0 & 3\end{array}$ & $p$-value & 0.241 & 0.210 & 0.832 & 0.881 & 0.954 & 0.446 & 0.290 \\
\hline & & $\alpha$ & $\left.\begin{array}{l}2 \\
1\end{array}\right]$ & Power & 0.382 & 0.374 & 0.494 & 0.464 & 0.570 & 0.106 & 0.607 \\
\hline \multirow{2}{*}{9} & 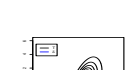 & $\Omega$ & $\begin{array}{ll}2 & 1 \\
1 & 3\end{array}$ & $p$-value & 0.367 & 0.395 & 0.735 & 0.879 & 0.844 & 0.211 & 0.266 \\
\hline & & $\alpha$ & $\begin{array}{r}2 \\
-1\end{array}$ & Power & 0.548 & 0.551 & 0.504 & 0.589 & 0.592 & 0.366 & 0.616 \\
\hline \multirow{2}{*}{10} & 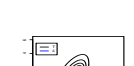 & $\Omega$ & $\begin{array}{ll}2 & 1 \\
1 & 3\end{array}$ & $p$-value & 0.402 & 0.392 & 0.708 & 0.296 & 0.166 & 0.223 & 0.277 \\
\hline & & $\alpha$ & $\left.\begin{array}{r}-2 \\
1\end{array}\right]$ & Power & 0.887 & 0.885 & 0.495 & 0.477 & 0.421 & 0.628 & 0.505 \\
\hline \multirow{2}{*}{11} & 国 & $\Omega$ & $\begin{array}{ll}2 & 0 \\
0 & 3\end{array}$ & $p$-value & 0.806 & 0.808 & 0.757 & 0.885 & 0.902 & 0.382 & 0.279 \\
\hline & & $\alpha$ & $\left.\begin{array}{l}1 \\
0\end{array}\right]$ & Power & 0.867 & 0.863 & 0.449 & 0.544 & 0.550 & 0.675 & 0.498 \\
\hline \multirow{2}{*}{12} & & $\Omega$ & $\begin{array}{r}2 \\
-1\end{array}$ & $p$-value & 0.000 & 0.000 & 0.000 & 0.000 & 0.000 & 0.000 & 0.000 \\
\hline & & $\alpha$ & $\left.\begin{array}{l}0 \\
0\end{array}\right]$ & Power & 0.000 & 0.000 & 0.000 & 0.000 & 0.000 & 0.000 & 0.000 \\
\hline
\end{tabular}


Table 12: $p$-value and power for test for bivariate $t$ distribution against skew- $t$ distribution (2)

\begin{tabular}{|c|c|c|c|c|c|c|c|c|c|c|}
\hline \multirow{2}{*}{$\#$} & \multirow{2}{*}{ Contour plot } & \multirow{2}{*}{\multicolumn{3}{|c|}{ Parameters }} & \multirow{2}{*}{$\begin{array}{c}\text { Srivastava } \\
\beta_{1 p}^{2} \\
\end{array}$} & \multicolumn{2}{|c|}{ Mori-Rohatgi-Szekely } & \multicolumn{2}{|c|}{ Kollo } & \multirow{2}{*}{$\begin{array}{l}\text { Song } \\
S(f) \\
\end{array}$} \\
\hline & & & & & & $s(\mathbf{y})_{\text {sup }}$ & $s(\mathbf{y})_{\text {sum }}$ & $b(\mathbf{y})_{\text {sup }}$ & $b(\mathbf{y})_{\text {sum }}$ & \\
\hline \multirow{2}{*}{1} & & $\Omega$ & $\left.\begin{array}{ll}2 & 1 \\
1 & 3\end{array}\right]$ & $p$-value & 0.321 & 0.366 & 0.314 & 0.372 & 0.327 & 0.682 \\
\hline & & $\alpha$ & $\left.\begin{array}{l}2 \\
5\end{array}\right]$ & Power & 0.349 & 0.522 & 0.555 & 0.548 & 0.572 & 0.594 \\
\hline \multirow{2}{*}{2} & & $\Omega$ & $\begin{array}{ll}2 & 0 \\
0 & 3\end{array}$ & $p$-value & 0.677 & 0.637 & 0.779 & 0.764 & 0.799 & 0.284 \\
\hline & & $\alpha$ & $\left.\begin{array}{l}2 \\
5\end{array}\right]$ & Power & 0.330 & 0.158 & 0.316 & 0.344 & 0.374 & 0.760 \\
\hline \multirow{2}{*}{3} & & $\Omega$ & $\begin{array}{ll}2 & 0 \\
0 & 3\end{array}$ & $p$-value & 0.325 & 0.540 & 0.621 & 0.548 & 0.598 & 0.615 \\
\hline & & $\alpha$ & $\left.\begin{array}{l}0 \\
5\end{array}\right]$ & Power & 0.606 & 0.493 & 0.488 & 0.465 & 0.442 & 0.440 \\
\hline \multirow{2}{*}{4} & & $\Omega$ & $\begin{array}{ll}2 & 1 \\
1 & 3\end{array}$ & $p$-value & 0.132 & 0.354 & 0.386 & 0.358 & 0.384 & 0.595 \\
\hline & & $\alpha$ & $\left.\begin{array}{l}0 \\
5\end{array}\right]$ & Power & 0.698 & 0.813 & 0.832 & 0.835 & 0.842 & 0.364 \\
\hline \multirow{2}{*}{5} & & $\Omega$ & $\begin{array}{ll}2 & 1 \\
1 & 3\end{array}$ & $p$-value & 0.217 & 0.520 & 0.563 & 0.536 & 0.574 & 0.725 \\
\hline & & $\alpha$ & $\left.\begin{array}{l}2 \\
3\end{array}\right]$ & Power & 0.486 & 0.424 & 0.393 & 0.357 & 0.355 & 0.444 \\
\hline \multirow{2}{*}{6} & 国 & $\Omega$ & $\begin{array}{ll}2 & 0 \\
0 & 3\end{array}$ & $p$-value & 0.562 & 0.698 & 0.841 & 0.818 & 0.873 & 0.659 \\
\hline & & $\alpha$ & $\left.\begin{array}{r}2 \\
-3\end{array}\right]$ & Power & 0.642 & 0.742 & 0.470 & 0.721 & 0.458 & 0.376 \\
\hline \multirow{2}{*}{7} & 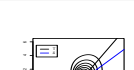 & $\Omega$ & $\begin{array}{ll}2 & 1 \\
1 & 3\end{array}$ & $p$-value & 0.497 & 0.260 & 0.287 & 0.272 & 0.281 & 0.468 \\
\hline & & $\alpha$ & $\left.\begin{array}{l}2 \\
1\end{array}\right]$ & Power & 0.734 & 0.804 & 0.797 & 0.797 & 0.793 & 0.248 \\
\hline \multirow{2}{*}{8} & $\Xi$ & $\Omega$ & $\begin{array}{ll}2 & 0 \\
0 & 3\end{array}$ & $p$-value & 0.201 & 0.335 & 0.471 & 0.347 & 0.449 & 0.738 \\
\hline & & $\alpha$ & $\left.\begin{array}{l}2 \\
1\end{array}\right]$ & Power & 0.394 & 0.474 & 0.525 & 0.489 & 0.460 & 0.585 \\
\hline \multirow{2}{*}{9} & 国 & $\Omega$ & $\begin{array}{ll}2 & 1 \\
1 & 3\end{array}$ & $p$-value & 0.424 & 0.219 & 0.252 & 0.110 & 0.242 & 0.704 \\
\hline & & $\alpha$ & $\left.\begin{array}{r}2 \\
-1\end{array}\right]$ & Power & 0.567 & 0.669 & 0.707 & 0.683 & 0.692 & 0.575 \\
\hline \multirow{2}{*}{10} & 国 & $\Omega$ & $\begin{array}{ll}2 & 1 \\
1 & 3\end{array}$ & $p$-value & 0.407 & 0.263 & 0.325 & 0.263 & 0.314 & 0.636 \\
\hline & & $\alpha$ & $\left.\begin{array}{r}-2 \\
1\end{array}\right]$ & Power & 0.887 & 0.187 & 0.131 & 0.120 & 0.121 & 0.103 \\
\hline \multirow{2}{*}{11} & $\Xi$ & $\Omega$ & $\begin{array}{ll}2 & 0 \\
0 & 3\end{array}$ & $p$-value & 0.803 & 0.760 & 0.302 & 0.280 & 0.303 & 0.222 \\
\hline & & $\alpha$ & $\begin{array}{l}1 \\
0\end{array}$ & Power & 0.860 & 0.896 & 0.871 & 0.852 & 0.855 & 0.114 \\
\hline \multirow{2}{*}{12} & & $\Omega$ & $\begin{array}{rr}2 & - \\
-1 & \end{array}$ & $p$-value & 0.000 & 0.000 & 0.000 & 0.000 & 0.000 & 0.000 \\
\hline & & $\alpha$ & $\left.\begin{array}{l}0 \\
0\end{array}\right]$ & Power & 0.000 & 0.000 & 0.000 & 0.000 & 0.000 & 0.000 \\
\hline
\end{tabular}


Table 13: $p$-value and power for test for trivariate $t$ distribution against skew- $t$ distribution (1)

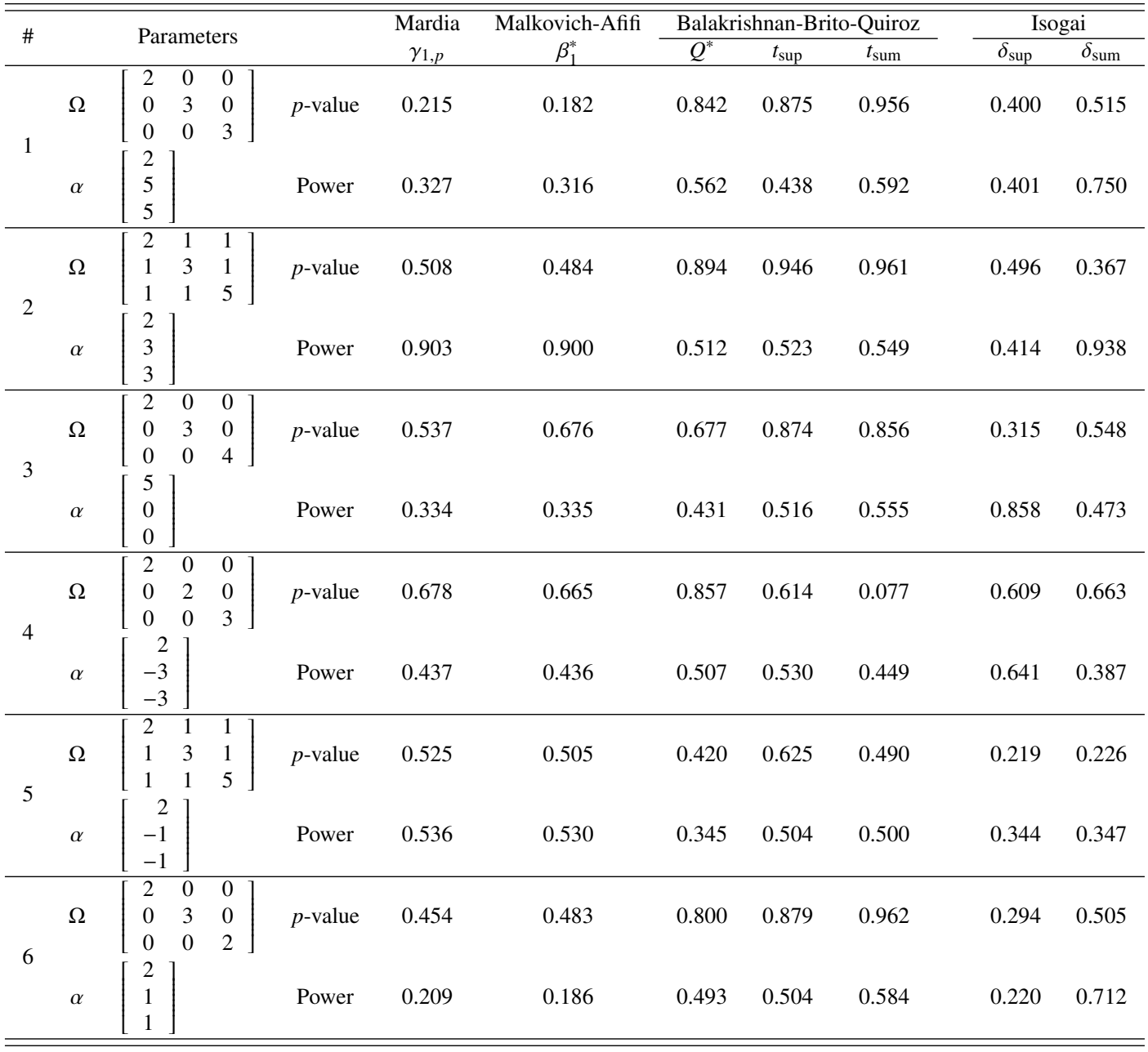

(2014), these two variables are well fitted by bivariate skew- $t$ distribution. Table 15 shows the maximum likelihood estimates of parameters in skew- $t$ distribution. Figure 1 displays the scatter plot for the two variables with the counter lines of the fitted distribution and Figure 2 illustrates goodness-offit. Table 16 provides all multivariate skewness measures for the fitted skew- $t$ distribution.

\section{Discussion}

Multivariate skewness measures are derived for the scale mixtures of skew-normal distributions. The case of multivariate skew- $t$ distribution is considered in detail. The comparison among the measures is explored. Furthermore the similarities and differences of the skewness measures between skewnormal and skew- $t$ distributions are also described. Performance of the measures and comparisons are done using $p$-values and powers. One real data application is also added. We plan to extend the multivariate skewness measures to multivariate kurtosis measures in an additional study. 
Table 14: $p$-value and power for test for trivariate $t$ distribution against skew- $t$ distribution (2)

\begin{tabular}{|c|c|c|c|c|c|c|c|c|c|c|}
\hline \multirow{2}{*}{ \# } & & \multirow{2}{*}{\multicolumn{2}{|c|}{ Parameters }} & & \multirow{2}{*}{$\begin{array}{c}\text { Srivastava } \\
\beta_{1 p}^{2} \\
\end{array}$} & \multicolumn{2}{|c|}{ Mori-Rohatgi-Szekely } & \multicolumn{2}{|c|}{ Kollo } & \multirow{2}{*}{$\begin{array}{l}\text { Song } \\
S(f)\end{array}$} \\
\hline & & & & & & $s(\mathbf{y})_{\text {sup }}$ & $s(\mathbf{y})_{\text {sum }}$ & $b(\mathbf{y})_{\text {sup }}$ & $b(\mathbf{y})_{\text {sum }}$ & \\
\hline \multirow{5}{*}{1} & & 20 & & & & & & & & \\
\hline & $\Omega$ & 03 & 0 & $p$-value & 0.205 & 0.403 & 0.385 & 0.354 & 0.416 & 0.782 \\
\hline & & $0 \quad 0$ & 3 & & & & & & & \\
\hline & $\alpha$ & $\begin{array}{l}2 \\
5\end{array}$ & & Power & 0347 & 0324 & 0342 & 0357 & 0381 & 0639 \\
\hline & & 5 & & ( & ו & 0.024 & 0.542 & 0.531 & 0.381 & 0.039 \\
\hline \multirow{5}{*}{2} & & $\begin{array}{ll}2 & 1\end{array}$ & & & & & & & & \\
\hline & $\Omega$ & 13 & 1 & $p$-value & 0.508 & 0.346 & 0.303 & 0.345 & 0.316 & 0.47 \\
\hline & & 11 & 5 & & & & & & & \\
\hline & $\alpha$ & $\left.\begin{array}{l}2 \\
3\end{array}\right]$ & & & 0898 & 0911 & 0.932 & 0.931 & 0.951 & 0110 \\
\hline & $\alpha$ & $\begin{array}{l}5 \\
3\end{array}$ & & rower & 0.898 & 0.911 & 0.932 & 0.931 & 0.951 & 0.110 \\
\hline \multirow{4}{*}{3} & & 20 & & & & & & & & \\
\hline & $\Omega$ & 03 & 0 & $p$-value & 0.736 & 0.933 & 0.932 & 0.929 & 0.910 & 0.810 \\
\hline & & 00 & 4 & & & & & & & \\
\hline & & 0 & & $10 \mathrm{vat}$ & 0.50 & 0.203 & נסדיד ט & 0.212 & 0.752 & ד \\
\hline \multirow{5}{*}{4} & & 20 & 0 & & & & & & & \\
\hline & $\Omega$ & 02 & 0 & $p$-value & 0.587 & 0.520 & 0.729 & 0.638 & 0.708 & 0.262 \\
\hline & & $0 \quad 0$ & 3 & & & & & & & \\
\hline & $\alpha$ & -3 & & Power & 0.295 & 0.634 & 0.404 & 0.429 & 0.429 & 0.595 \\
\hline & & $-3\rfloor$ & & & & & & & & \\
\hline \multirow{5}{*}{5} & & $\begin{array}{ll}2 & 1\end{array}$ & 1 & & & & & & & \\
\hline & $\Omega$ & 13 & 1 & $p$-value & 0.539 & 0.227 & 0.220 & 0.251 & 0.246 & 0.464 \\
\hline & & 11 & 5 & & & & & & & \\
\hline & $\alpha$ & -1 & & Power & 0.593 & 0.219 & 0.232 & 0.246 & 0.323 & 0.429 \\
\hline & & -1 & & & & & & & & \\
\hline \multirow{6}{*}{6} & & $\begin{array}{ll}2 & 0\end{array}$ & & & & & & & & \\
\hline & $\Omega$ & $0 \quad 3$ & 0 & $p$-value & 0.579 & 0.583 & 0.358 & 0.540 & 0.336 & 0.610 \\
\hline & & $0 \quad 0$ & & & & & & & & \\
\hline & & 2 & & & & & & & & \\
\hline & $\alpha$ & 1 & & Power & 0.155 & 0.368 & 0.425 & 0.482 & 0.483 & 0.752 \\
\hline & & 1 & & & & & & & & \\
\hline
\end{tabular}

Table 15: Parameter estimates for skew- $t$ distribution

\begin{tabular}{cccr}
\hline $\boldsymbol{\xi}$ & $v$ & $\boldsymbol{\Omega}$ & $\boldsymbol{\alpha}$ \\
\hline$\left[\begin{array}{r}0.1500 \\
-1.0857\end{array}\right]$ & 14.9274 & {$\left[\begin{array}{rr}0.8974 & -0.0308 \\
-0.0308 & 1.8577\end{array}\right]$} & {$\left[\begin{array}{l}0.8343 \\
4.0916\end{array}\right]$} \\
\hline \hline
\end{tabular}

Table 16: Skewness measures for fitted skew- $t$ distribution

\begin{tabular}{|c|c|c|c|c|}
\hline \multirow{2}{*}{$\begin{array}{c}\text { Mardia } \\
\gamma_{1, p}\end{array}$} & \multirow{2}{*}{$\begin{array}{c}\text { Malkovich-Afifi } \\
\beta_{1}^{*} \\
\end{array}$} & \multicolumn{2}{|c|}{ Balakrishnan-Brito-Quiroz } & \multirow{2}{*}{$\begin{array}{c}\text { Isogai } \\
\delta\end{array}$} \\
\hline & & $Q^{*}$ & $\mathbf{t}$ & \\
\hline 1.5030 & 1.1563 & 0.1512 & $\begin{array}{r}-0.0812 \\
0.3802\end{array}$ & $\begin{array}{r}-0.2161 \\
0.9533\end{array}$ \\
\hline $\begin{array}{c}\text { Srivastava } \\
\beta_{1 p}^{2}\end{array}$ & $\begin{array}{l}\text { Mori-Rohatgi-Szekely } \\
s(\mathbf{y})\end{array}$ & $\begin{array}{c}\text { Kollo } \\
b(\mathbf{y})\end{array}$ & $\begin{array}{l}\text { Song } \\
S(f)\end{array}$ & \\
\hline 0.3533 & $\begin{array}{r}-0.2605 \\
1.1445 \\
\end{array}$ & $\begin{array}{l}0.0064 \\
0.7716 \\
\end{array}$ & 0.3736 & \\
\hline
\end{tabular}




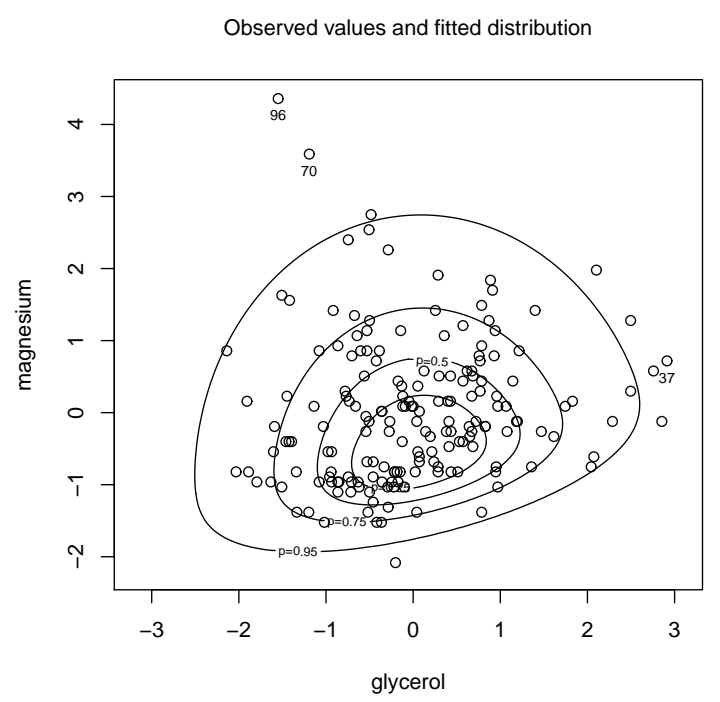

Figure 1: Contour plot of total phenols content of Grignolino.
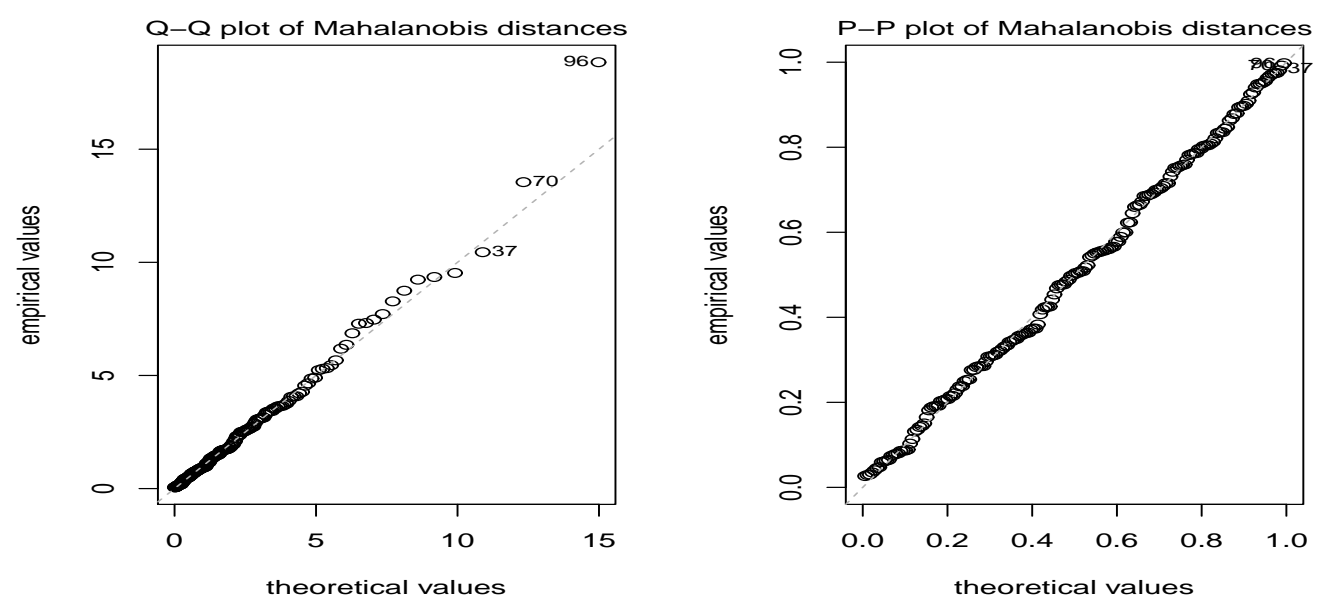

Figure 2: $Q-Q$ and P-P plots of total phenols content of Grignolino.

\section{Acknowledgments}

Kim's research was supported by Basic Science Research Program through the National Research Foundation of Korea (NRF) funded by the Ministry of Education (2015R1D1A1A01059161). This paper was written as part of Konkuk University's research support program for its faculty on sabbatical leave in 2016.

\section{References}

Arevalillo JM and Navarro H (2015). A note on the direction maximizing skewness in multivariate skew- $t$ vectors, Statistics and Probability Letters, 96, 328-332. 
Azzalini A and Capitanio A (2014). The Skew-Normal and Related Families, Cambridge, United Kingdom.

Balakrishnan N, Brito MR, and Quiroz AJ (2007). A vectorial notion of skewness and its use in testing for multivariate symmetry, Communications in Statistics Theory and Methods, 36, 1757-1767.

Balakrishnan N and Scarpa B (2012). Multivariate measures of skewness for the skew-normal distribution, Journal of Multivariate Analysis, 104, 73-87.

Benjamini Y and Krieger AM (2006). Skewness: Concepts and Measures. In Kotz S, Balakrishnan N, Read CB, Vidakovic B (eds), Encyclopedia of Statistical Sciences (2nd ed), John Wiley \& Sons, Hoboken, New Jersey.

Branco MD and Dey DK (2001). A general class of multivariate skew elliptical distributions, Journal of Multivariate Analysis, 79, 99-113.

Capitanio A (2012). On the canonical form of scale mixtures of skew-normal distributions. Available on arXiv (http://arxiv.org/abs/1207.0797).

Isogai T (1982). On a measure of multivariate skewness and a test for multivariate normality, Annals of the Institute of Statistical Mathematics, 34A, 531-541.

Kim HM and Kim C (2017). Moments of scale mixtures of skew-normal distributions and their quadratic forms, Communications in Statistics Theory and Methods, 46, 1117-1126.

Kollo T (2008). Multivariate skewness and kurtosis measures with an application in ICA, Journal of Multivariate Analysis, 99, 2328-2338.

Malkovich JF and Afifi AA (1973). On tests for multivariate normality, Journal of the American Statistical Association, 68, 176-179.

Mardia KV (1970). Measures of multivariate skewness and kurtosis with applications, Biometrika, 36, 519-530.

Móri TF, Rohatgi VK, and Székely GJ (1993). On multivariate skewness and kurtosis, Theory of Probability and its Applications, 38, 547-551.

Song KS (2001). Renyi information, loglikelihood and an intrinsic distribution measure, Journal of Statistical Planning and Inference, 93, 51-69.

Srivastava MS (1984). A measure of skewness and kurtosis and a graphical method for assessing multivariate normality, Statistics \& Probability Letters, 2, 263-267 